\title{
Semidefinite Relaxation of Quadratic Optimization Problems
}

\author{
Zhi-Quan Luo, Wing-Kin Ma, Anthony Man-Cho So, Yinyu Ye, and Shuzhong Zhang
}

\section{INTRODUCTION}

In recent years, the semidefinite relaxation (SDR) technique has been at the center of some of the very exciting developments in the area of signal processing and communications, and it has shown great significance and relevance on a variety of applications. Roughly speaking, SDR is a powerful, computationally efficient approximation technique for a host of very difficult optimization problems. In particular, it can be applied to many nonconvex quadratically constrained quadratic programs (QCQPs) in an almost mechanical fashion. These include the following problems:

$$
\begin{aligned}
\min _{\boldsymbol{x} \in \mathbb{R}^{n}} & \boldsymbol{x}^{T} \boldsymbol{C} \boldsymbol{x} \\
\mathrm{s.t.} & \boldsymbol{x}^{T} \boldsymbol{F}_{i} \boldsymbol{x} \geq g_{i}, \quad i=1, \ldots, p, \\
& \boldsymbol{x}^{T} \boldsymbol{H}_{i} \boldsymbol{x}=l_{i}, \quad i=1, \ldots, q,
\end{aligned}
$$

where the given matrices $\boldsymbol{C}, \boldsymbol{F}_{1}, \ldots, \boldsymbol{F}_{p}, \boldsymbol{H}_{1}, \ldots, \boldsymbol{H}_{q}$ are assumed to be general real symmetric matrices, possibly indefinite. The class of nonconvex QCQPs (1) captures many problems that are of interest to the signal processing and communications community. For instance, consider the Boolean quadratic program (BQP)

$$
\begin{aligned}
\min _{\boldsymbol{x} \in \mathbb{R}^{n}} & \boldsymbol{x}^{T} \boldsymbol{C} \boldsymbol{x} \\
\text { s.t. } & x_{i}^{2}=1, \quad i=1, \ldots, n .
\end{aligned}
$$

The BQP is long-known to be a computationally difficult problem. In particular, it belongs to the class of NP-hard problems. Nevertheless, being able to handle the BQP well has an enormous impact on multiple-input-multiple-output (MIMO) detection and multiuser detection. Another important yet NP-hard problem in the nonconvex QCQP class (1) is

$$
\begin{aligned}
& \min _{\boldsymbol{x} \in \mathbb{R}^{n}} \boldsymbol{x}^{T} \boldsymbol{C} \boldsymbol{x} \\
& \text { s.t. } \quad \boldsymbol{x}^{T} \boldsymbol{F}_{i} \boldsymbol{x} \geq 1, \quad i=1, \ldots, m,
\end{aligned}
$$

where $\boldsymbol{C}, \boldsymbol{F}_{1}, \ldots, \boldsymbol{F}_{m}$ are all positive semidefinite. Problem (3) captures the multicast downlink transmit beamforming problem; see [1] for details. An illustration of an instance of Problem (3) is provided in Fig. 1. As seen from the figure, the feasible set of (3) is the intersection of the exteriors of multiple ellipsoids, which makes the problem difficult.

As a matter of fact, SDR has been studied and applied in the optimization community long before it made its impact on signal processing and communications. The idea of SDR can already be found in an early paper of Lovász in 1979 [2], but it was arguably the seminal work of Goemans and Williamson in 1995 [3] that sparked the significant interest in and rapid development of SDR techniques. In that work, it was shown that SDR can be used to provide an approximation

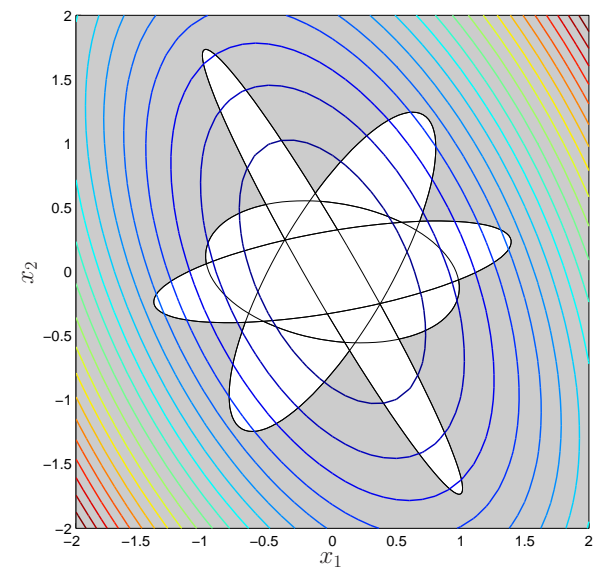

Fig. 1. A nonconvex QCQP in $\mathbb{R}^{2}$. Colored lines: contour of the objective function; gray area: the feasible set; black lines: boundary of each constraint.

accuracy of no worse than 0.8756 for the Maximum Cut problem (the BQP with some conditions on $C$ ). In other words, even though the Maximum Cut problem is NP-hard, one could efficiently obtain a solution whose objective value is at least 0.8756 times the optimal value using SDR. Since then, we have seen a number of dedicated theoretical analyses that establish the SDR approximation accuracy under different problem settings [3]-[11], and that have greatly improved our understanding of the capabilities of SDR. Today, we are even able to pin down a number of conditions under which SDR provides an exact optimal solution to the original problem [7], [12]-[16].

In the field of signal processing and communications, the introduction of SDR since the early 2000's has reshaped the way we see many topics today. Many practical experiences have already indicated that SDR is capable of providing accurate (and sometimes near-optimal) approximations. For instance, in MIMO detection, SDR is now known as an efficient highperformance approach [17]-[23] (see also [24]-[26] for blind MIMO detection). The promising empirical approximation performance of SDR has motivated new endeavors, leading to the creation of new research trends in some cases. One such example is in the area of transmit beamforming, which has attracted much recent interest; for a review of this exciting topic, please see the article by Gershman $e t$ al. in this special issue [1], and [27]. The effectiveness of transmit beamforming depends much on how well one can handle (often nonconvex) QCQPs, and its technical progress could have been slower if SDR had not been known to the signal processing community. Another example worth mentioning is sensor network 
localization, a practically important but technically challenging problem. SDR has proven to be an effective technique for tackling the sensor network localization problem, both in theory and practice [28]-[31]. In addition to the three major applications mentioned above, there are many other different applications of SDR, such as waveform design in radar [32], [33], phase unwrapping [34], robust blind beamforming [35], large-margin parameter estimation in speech recognition (see the article by Jiang and $\mathrm{Li}$ in this special issue [36] for further details), transmit $B_{1}$ shim in MRI [37], and many more [38][41]. It is anticipated that SDR would find more applications in the near future.

This paper aims to give an overview of SDR, with an emphasis on showing the underlying intuitions and various applications of this powerful tool. In fact, we will soon see that the implementation of SDR can be very easy, and that allows signal processing practitioners to quickly test the viability of SDR in their applications. Several highly successful applications will be showcased as examples. We will also endeavor to touch on some advanced, key theoretical results by highlighting their practical impacts and implications.

This paper is organized as follows. Section II describes the basic ideas of SDR and its operations. Section III showcases an SDR application, namely, MIMO detection. In Section IV we shed light into the randomization concept, which plays an indispensable role in both theoretical and practical advances of SDR. Section V considers extensions of SDR to more general cases. This is immediately followed by Section VI, where another application example, B1-shimming in MRI, is demonstrated. Section VII presents a theoretical subject, namely SDR rank reduction, which has important implications for the tightness of SDR approximation. Section VIII describes the application of SDR in sensor network localization. We draw conclusions and discuss further issues in Section IX.

\section{The Concept of SEMidefinite Relaxation}

To make the notation more concise, let us write our problem of interest-namely, the real-valued homogeneous QCQP in (1)_as follows:

$$
\begin{aligned}
\min _{\boldsymbol{x} \in \mathbb{R}^{n}} & \boldsymbol{x}^{T} \boldsymbol{C} \boldsymbol{x} \\
\text { s.t. } & \boldsymbol{x}^{T} \boldsymbol{A}_{i} \boldsymbol{x} \unrhd_{i} b_{i}, \quad i=1, \ldots, m .
\end{aligned}
$$

Here, ' $\unrhd_{i}$ ' can represent either ' $\geq$ ', '=', or ' $\leq$ ' for each $i$; and $\boldsymbol{C}, \boldsymbol{A}_{1}, \ldots, \boldsymbol{A}_{m} \in \mathbb{S}^{n}$, where $\mathbb{S}^{n}$ denotes the set of all real symmetric $n \times n$ matrices; and $b_{1}, \ldots, b_{m} \in \mathbb{R}$. A crucial first step in deriving an SDR of Problem (4) is to observe that

$$
\begin{aligned}
& \boldsymbol{x}^{T} \boldsymbol{C} \boldsymbol{x}=\operatorname{Tr}\left(\boldsymbol{x}^{T} \boldsymbol{C} \boldsymbol{x}\right)=\operatorname{Tr}\left(\boldsymbol{C} \boldsymbol{x} \boldsymbol{x}^{T}\right) \\
& \boldsymbol{x}^{T} \boldsymbol{A}_{i} \boldsymbol{x}=\operatorname{Tr}\left(\boldsymbol{x}^{T} \boldsymbol{A}_{i} \boldsymbol{x}\right)=\operatorname{Tr}\left(\boldsymbol{A}_{i} \boldsymbol{x} \boldsymbol{x}^{T}\right) .
\end{aligned}
$$

In particular, both the objective function and constraints in (4) are linear in the matrix $\boldsymbol{x} \boldsymbol{x}^{T}$. Thus, by introducing a new variable $\boldsymbol{X}=\boldsymbol{x} \boldsymbol{x}^{T}$ and noting that $\boldsymbol{X}=\boldsymbol{x} \boldsymbol{x}^{T}$ is equivalent to $\boldsymbol{X}$ being a rank one symmetric positive semidefinite (PSD) matrix, we obtain the following equivalent formulation of
Problem (4):

$$
\begin{aligned}
\min _{\boldsymbol{X} \in \mathbb{S}^{n}} & \operatorname{Tr}(\boldsymbol{C} \boldsymbol{X}) \\
\text { s.t. } & \operatorname{Tr}\left(\boldsymbol{A}_{i} \boldsymbol{X}\right) \unrhd_{i} b_{i}, \quad i=1, \ldots, m, \\
& \boldsymbol{X} \succeq \mathbf{0}, \quad \operatorname{rank}(\boldsymbol{X})=1 .
\end{aligned}
$$

Here, we use $\boldsymbol{X} \succeq \mathbf{0}$ to indicate that $\boldsymbol{X}$ is PSD.

At this point, it may seem that we have not achieved much, as Problem (5) is just as difficult to solve as Problem (4). However, the formulation in (5) allows us to identify the fundamental difficulty in solving Problem (4). Indeed, the only difficult constraint in (5) is the rank constraint $\operatorname{rank}(\boldsymbol{X})=1$, which is nonconvex (the objective function and all other constraints are convex in $\boldsymbol{X}$ ). Thus, we may as well drop it to obtain the following relaxed version of Problem (4):

$$
\begin{aligned}
\min _{\boldsymbol{X} \in \mathbb{S}^{n}} & \operatorname{Tr}(\boldsymbol{C} \boldsymbol{X}) \\
\text { s.t. } & \operatorname{Tr}\left(\boldsymbol{A}_{i} \boldsymbol{X}\right) \unrhd_{i} b_{i}, \quad i=1, \ldots, m, \\
& \boldsymbol{X} \succeq \mathbf{0} .
\end{aligned}
$$

Problem (6) is known as an SDR of Problem (4), where the name stems from the fact that (6) is an instance of semidefinite programming (SDP). The upshot of the formulation in (6) is that it can be solved, to any arbitrary accuracy, in a numerically reliable and efficient fashion. In fact, SDRs can now be handled very conveniently and effectively by readily available (and free) software packages. Let us give an example: Suppose that ' $\unrhd_{i}$ ' equal ' $\geq$ ' for $i=1, \ldots, p$, and ' $\unrhd_{i}$ ' equal '=' for $i=p+1, \ldots, m$. Using the convex optimization toolbox CVX [42], we can solve (6) in MATLAB with the following piece of code:

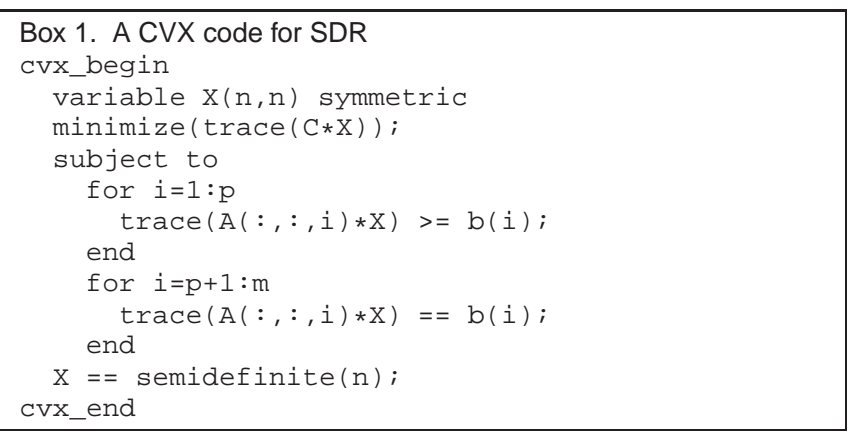

While advances in convex optimization and software have enabled us to solve SDPs easily and transparently, one might question how effective is the process (how fast or slow it would be?). In the backstage most convex optimization toolboxes handle SDPs using an interior-point algorithm, a sophisticated topic in its own right (see, e.g., [43]). Simply speaking, the SDR problem (6) can be solved with a worst case complexity of

$$
\mathcal{O}\left(\max \{m, n\}^{4} n^{1 / 2} \log (1 / \epsilon)\right)
$$

given a solution accuracy $\epsilon>0{ }^{1}$. The complexity above does not assume sparsity or any special structures in the data matrices $\boldsymbol{C}, \boldsymbol{A}_{1}, \ldots, \boldsymbol{A}_{m}$. Some algorithms, such as SeDuMi [46]

\footnotetext{
${ }^{1}$ Our reported complexity order is obtained by counting the arithmetic operations of a specific interior-point method, namely the primal-dual pathfollowing method in [44]. See [45] for a more detailed description on the operation count.
} 
(employed as one of the core solvers in CVX), can utilize data matrix sparsity to speed up the solution process. We also refer the readers to the article [47] in this special issue for other fast real-time convex optimization solvers. For certain specially structured SDR problems, one can even exploit the problem structures to build fast customized interior-point algorithms. For example, for BQP, a custom-built interiorpoint algorithm [44] can solve SDR with a complexity of $\mathcal{O}\left(n^{3.5} \log (1 / \epsilon)\right)$ [instead of $\left.\mathcal{O}\left(n^{4.5} \log (1 / \epsilon)\right)\right]$. Furthermore, the SDR complexity scales slowly (logarithmically) with $\epsilon$ and most applications do not require a very high solution precision. Hence, simply speaking, we can say that

SDR is a computationally efficient approximation approach to QCQP, in the sense that its complexity is polynomial in the problem size $n$ and the number of constraints $m$.

Of course, there is no free lunch in turning the NP-hard Problem (4) (which is equivalent to Problem (5)) into the polynomial-time solvable Problem (6). Indeed, a fundamental issue that one must address when using SDR is how to convert a globally optimal solution $\boldsymbol{X}^{\star}$ to Problem (6) into a feasible solution $\tilde{\boldsymbol{x}}$ to Problem (4). Now, if $\boldsymbol{X}^{\star}$ is of rank one, then there is nothing to do, for we can write $\boldsymbol{X}^{\star}=\boldsymbol{x}^{\star} \boldsymbol{x}^{\star T}$, and $\boldsymbol{x}^{\star}$ will be a feasible — and in fact optimal-solution to Problem (4). On the other hand, if the rank of $\boldsymbol{X}^{\star}$ is larger than 1 , then we must somehow extract from it, in an efficient manner, a vector $\tilde{\boldsymbol{x}}$ that is feasible for Problem (4). There are many ways to do this, and they generally follow some intuitively reasonable heuristics (true even in engineering sense). However, we must emphasize that even though the extracted solution is feasible for Problem (4), it is in general not an optimal solution (for otherwise we would have solved an NP-hard problem in polynomial time).

As an illustration, consider the intuitively appealing idea of applying a rank-one approximation on $\boldsymbol{X}^{\star}$. Specifically, let $r=\operatorname{rank}\left(\boldsymbol{X}^{\star}\right)$, and let

$$
\boldsymbol{X}^{\star}=\sum_{i=1}^{r} \lambda_{i} \boldsymbol{q}_{i} \boldsymbol{q}_{i}^{T}
$$

denote the eigen-decomposition of $\boldsymbol{X}^{\star}$, where $\lambda_{1} \geq \lambda_{2} \geq$ $\ldots \geq \lambda_{r}>0$ are the eigenvalues and $\boldsymbol{q}_{1}, \ldots, \boldsymbol{q}_{r} \in \mathbb{R}^{n}$ are the respective eigenvectors. Since the best rank-one approximation $\boldsymbol{X}_{1}^{\star}$ to $\boldsymbol{X}^{\star}$ (in the least 2-norm sense) is given by $\boldsymbol{X}_{1}^{\star}=$ $\lambda_{1} \boldsymbol{q}_{1} \boldsymbol{q}_{1}^{T}$, we may define $\tilde{\boldsymbol{x}}=\sqrt{\lambda_{1}} \boldsymbol{q}_{1}$ as our candidate solution to Problem (4), provided that it is feasible. Otherwise, we can try to map $\tilde{\boldsymbol{x}}$ to a "nearby" feasible solution $\hat{\boldsymbol{x}}$ of Problem (4). In general, such a mapping is problem dependent, but it can be quite simple. For example, for the BQP (2) where $x_{i}^{2}=1$ for all $i$, we can obtain a feasible solution from $\tilde{\boldsymbol{x}}$ via $\hat{\boldsymbol{x}}=\operatorname{sgn}(\tilde{\boldsymbol{x}})$, where $\operatorname{sgn}(\cdot)$ is the element-wise signum function.

Our basic description of SDR is now complete. Before we proceed, some remarks are in order.

1) Now that we have seen one method of extracting a feasible solution $\hat{\boldsymbol{x}}$ to Problem (4) from a solution $\boldsymbol{X}^{\star}$ to the SDP (6), it is natural to ask what is the quality of the extracted solution $\hat{x}$. It turns out that there are several measures available to address this issue. Although we will not discuss them at this point, it should be emphasized that regardless of which measure we use, the quality will certainly depend on the method by which we extract the solution $\hat{x}$.

2) Apart from the rank relaxation interpretation of SDR as described above, there is another interpretation that is based on Lagrangian duality. Specifically, it can be shown that the SDR (6) is a Lagrangian bidual of the original problem (4). We refer the reader to, e.g., [48] for details.

\section{Applichtion: MiMo Detection}

Let us show an example of SDR application before proceeding to further advanced concepts and applications.

The problem we consider is MIMO detection, a frequently encountered problem in digital communications. To put it into context, consider a generic $N$-input $M$-output model

$$
\boldsymbol{y}_{C}=\boldsymbol{H}_{C} \boldsymbol{s}_{C}+\boldsymbol{v}_{C} .
$$

Here, $\boldsymbol{y}_{C} \in \mathbb{C}^{M}$ is the received vector, $\boldsymbol{H}_{C} \in \mathbb{C}^{M \times N}$ is the MIMO channel, $s_{C} \in \mathbb{C}^{N}$ is the transmitted symbol vector, and $\boldsymbol{v}_{C} \in \mathbb{C}^{M}$ is an additive white Gaussian noise vector. Eq. (7) is popularly used to model point-to-point multiple-antenna systems such as the spatial multiplexing (or V-BLAST) depicted in Fig. 2. In fact, it is known (see, e.g., [49]) that the same model as in (7) can be used to formulate detection problems in many other communication scenarios, such as multiuser systems, space-time coding systems, spacefrequency coding systems, and combinations such as multiuser multi-antenna systems. The wide applicability of the MIMO model (7) makes its respective detection problem attractive and important to tackle.

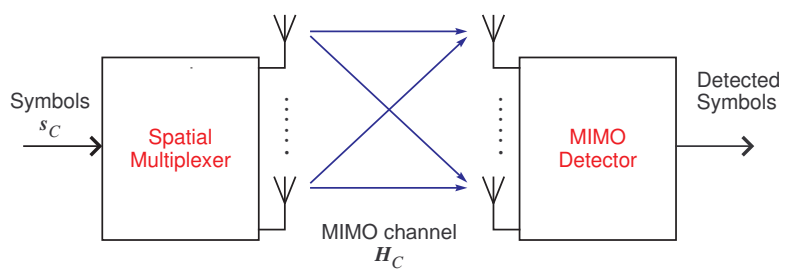

Fig. 2. The spatial multiplexing system.

In this application example we assume that the transmitted symbols follow a quaternary phase-shift-keying (QPSK) constellation; i.e., $s_{C, i} \in\{ \pm 1 \pm j\}$ for all $i$. We are interested in the maximum-likelihood (ML) MIMO detection, which is optimal in yielding the minimum error probability of detecting $s_{C}$. It can be shown that the ML problem is equivalent to the discrete least squares problem

$$
\min _{\boldsymbol{s}_{C} \in\{ \pm 1 \pm j\}^{N}}\left\|\boldsymbol{y}_{C}-\boldsymbol{H}_{C} \boldsymbol{s}_{C}\right\|^{2},
$$

which is NP-hard [50]. Recent advances in MIMO detection have provided a practically efficient way of finding a globally optimal ML solution; viz., the sphere decoding methods [49]. 
Sphere decoding has been found to be computationally fast for small to moderate problem sizes; e.g., $N \leq 20$. However, it has been proven that the complexity of sphere decoding is exponential in $N$ even in an average sense [51].

On the other hand, SDR can be used to produce an approximate solution to the ML MIMO detection problem in $\mathcal{O}\left(N^{3.5}\right)$ time, which is polynomial in $N$. The trick is to turn (8) into a real-valued homogeneous QCQP. Indeed, by letting

$\boldsymbol{y}=\left[\begin{array}{c}\Re\left\{\boldsymbol{y}_{C}\right\} \\ \Im\left\{\boldsymbol{y}_{C}\right\}\end{array}\right], \boldsymbol{s}=\left[\begin{array}{c}\Re\left\{\boldsymbol{s}_{C}\right\} \\ \Im\left\{\boldsymbol{s}_{C}\right\}\end{array}\right], \boldsymbol{H}=\left[\begin{array}{cc}\Re\left\{\boldsymbol{H}_{C}\right\} & -\Im\left\{\boldsymbol{H}_{C}\right\} \\ \Im\left\{\boldsymbol{H}_{C}\right\} & \Re\left\{\boldsymbol{H}_{C}\right\}\end{array}\right]$,

we can rewrite (8) as the following real-valued problem:

$$
\min _{\boldsymbol{s} \in\{ \pm 1\}^{2 N}}\|\boldsymbol{y}-\boldsymbol{H} \boldsymbol{s}\|^{2} .
$$

Problem (9) is not a homogeneous QCQP, but we can homogenize it as follows:

$$
\begin{aligned}
\min _{\boldsymbol{s} \in \mathbb{R}^{2 N}, t \in \mathbb{R}} & \|t \boldsymbol{y}-\boldsymbol{H} \boldsymbol{s}\|^{2} \\
\text { s.t. } & t^{2}=1, \quad s_{i}^{2}=1, \quad i=1, \ldots, 2 N .
\end{aligned}
$$

Problem (10) is equivalent to (9) in the following sense: if $\left(\boldsymbol{x}^{\star}, t^{\star}\right)$ is an optimal solution to (10), then $\boldsymbol{x}^{\star}$ (resp. $-\boldsymbol{x}^{\star}$ ) is an optimal solution to (9) when $t^{\star}=1$ (resp. $t^{\star}=-1$ ). With the introduction of the extra variable $t$, Problem (10) can then be expressed as a homogeneous QCQP:

$$
\begin{aligned}
\min _{\boldsymbol{s} \in \mathbb{R}^{2 N}, t \in \mathbb{R}} & {\left[\begin{array}{ll}
\boldsymbol{s}^{T} & t
\end{array}\right]\left[\begin{array}{cc}
\boldsymbol{H}^{T} \boldsymbol{H} & -\boldsymbol{H}^{T} \boldsymbol{y} \\
-\boldsymbol{y}^{T} \boldsymbol{H} & \|\boldsymbol{y}\|^{2}
\end{array}\right]\left[\begin{array}{l}
\boldsymbol{s} \\
t
\end{array}\right] } \\
\text { s.t. } & t^{2}=1, \quad s_{i}^{2}=1, \quad i=1, \ldots, 2 N .
\end{aligned}
$$

Subsequently, SDR can be applied.

We now show some simulation results to illustrate how well SDR performs in practice. The simulation follows a standard MIMO setting (see, e.g., [49]), with problem size $(M, N)=(40,40)$. Note that for such a problem size, sphere decoding is computationally too slow to run in practice. We tested other benchmarked MIMO detectors, such as the linear and decision-feedback detectors, and the lattice-reductionaided detectors. The results are plotted in Fig. 3. We can see that SDR provides near-optimal bit error probability, and gives notably better performance than other MIMO detectors under test.

In Fig. 3 two performance curves are provided for SDR. The one labeled 'SDR with rank-1 approx.' is the eigenvector approximation method described in the last section. While this method is already competitive in performance, the alternative 'SDR with randomization' is even more promising. The notion of randomization will be discussed in Section IV.

Next, we evaluate the computational complexities of the various MIMO detectors. The results are plotted in Fig. 4. Of particular interest is the comparison between SDR and optimal sphere decoding. We see that SDR maintains a polynomialtime complexity with respect to $N$. For sphere decoding, the complexity is attractive for small to moderate $N$, say $N \leq 16$, but it increases very significantly (exponentially) otherwise.

We conclude this section by pointing out the current advances of this SDR application. In essence, the promising performance of SDR MIMO detection in QPSK and binary PSK (BPSK) has stimulated much interest. That has resulted

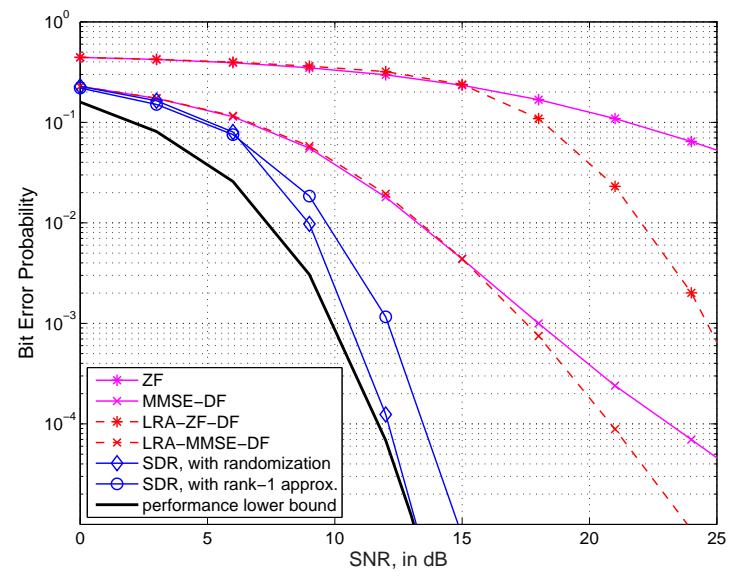

Fig. 3. Bit error probability performance of various MIMO detectors in a QPSK $40 \times 40$ MIMO system. 'ZF' - zero forcing, 'MMSE' - minimum mean square error, 'DF' — decision feedback, 'LRA' - lattice reduction aided. 'performance lower bound' is the bit error probability when no MIMO interference exists.

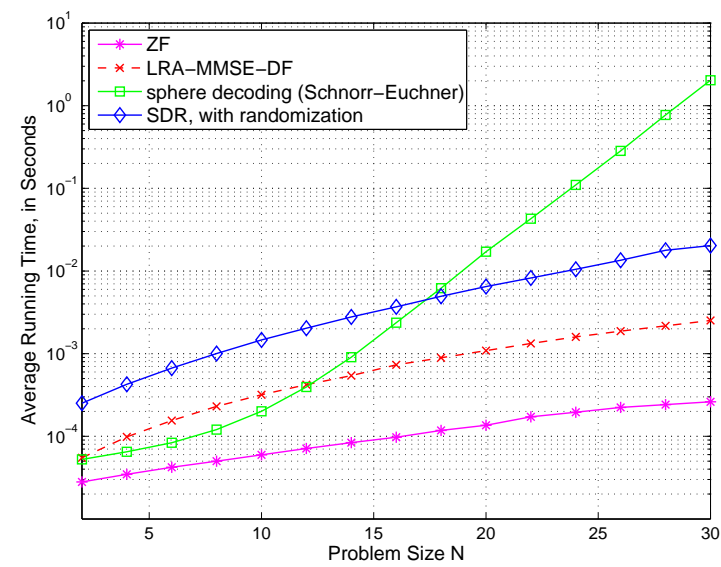

Fig. 4. Complexity comparison of various MIMO detectors. $\mathrm{SNR}=12 \mathrm{~dB}$.

in endeavors to extend SDR MIMO detection to other constellations, such as $M$-ary PSK [20] and $M$-ary QAM [22], [23], [52]-[55]. Moreover, treatments for coded MIMO systems [19], [56] and fast practical implementations [21], [45], [57] have been considered. On another front, the theoretical performance of SDR MIMO detection has been analyzed in various settings. For instance, it has been shown that SDR can achieve full receive diversity for BPSK [58]. Furthermore, SDR approximation accuracies relative to the true ML have been investigated in [59], [60].

\section{Randomization And Provable Approximation ACCURACIES}

Besides the eigenvector approximation method mentioned in Section II, randomization is another way to extract an approximate QCQP solution from an SDR solution $\boldsymbol{X}^{\star}$. The intuitive ideas behind randomization are not difficult to see, yet the theoretical implications that follow are far from trivialmany theoretical approximation accuracy results for SDRs are proven using randomization. To illustrate the main ideas, let 
us consider again the real-valued homogeneous QCQP

$$
\begin{aligned}
\min _{\boldsymbol{x} \in \mathbb{R}^{n}} & \boldsymbol{x}^{T} \boldsymbol{C} \boldsymbol{x} \\
\text { s.t. } & \boldsymbol{x}^{T} \boldsymbol{A}_{i} \boldsymbol{x} \unrhd_{i} b_{i}, \quad i=1, \ldots, m .
\end{aligned}
$$

Now, let $\boldsymbol{X} \in \mathbb{S}^{n}$ be an arbitrary symmetric positive semidefinite matrix. Consider a random vector $\boldsymbol{\xi} \in \mathbb{R}^{n}$ drawn according to the Gaussian distribution with zero mean and covariance $\boldsymbol{X}$; or $\boldsymbol{\xi} \sim \mathcal{N}(\mathbf{0}, \boldsymbol{X})$ for short. The intuition of randomization lies in considering the following stochastic QCQP:

$$
\begin{aligned}
& \min _{\boldsymbol{X} \in \mathbb{S}^{n},}, \mathrm{E}_{\boldsymbol{\boldsymbol { X }} \sim \mathcal{N}(\mathbf{0}, \boldsymbol{X})}\left\{\boldsymbol{\xi}^{T} \boldsymbol{C} \boldsymbol{\xi}\right\} \\
& \text { s.t. } \mathrm{E}_{\boldsymbol{\xi} \sim \mathcal{N}(\mathbf{0}, \boldsymbol{X})}\left\{\boldsymbol{\xi}^{T} \boldsymbol{A}_{i} \boldsymbol{\xi}\right\} \unrhd_{i} b_{i}, \quad i=1, \ldots, m,
\end{aligned}
$$

where we manipulate the covariance matrix of $\boldsymbol{\xi}$ so that the expected value of the quadratic objective is minimized and the quadratic constraints are satisfied in expectation. Interestingly, through the simple relation $\boldsymbol{X}=\mathrm{E}_{\boldsymbol{\xi} \sim \mathcal{N}(\mathbf{0}, \boldsymbol{X})}\left\{\boldsymbol{\xi} \boldsymbol{\xi}^{T}\right\}$, one can see that the stochastic QCQP in (13) is equivalent to the SDR

$$
\begin{aligned}
\min _{\boldsymbol{X} \in \mathbb{S}^{n}, \boldsymbol{X} \succeq \mathbf{0}} & \operatorname{Tr}(\boldsymbol{C X}) \\
\text { s.t. } & \operatorname{Tr}\left(\boldsymbol{A}_{i} \boldsymbol{X}\right) \unrhd_{i} b_{i}, \quad i=1, \ldots, m .
\end{aligned}
$$

Thus, the stochastic QCQP interpretation of SDR in (13) provides us with an alternative way to generate approximate solutions to the QCQP (12). Indeed, after obtaining an optimal solution $\boldsymbol{X}^{\star}$ to the SDP (14), we can generate a random vector $\boldsymbol{\xi} \sim \mathcal{N}\left(\mathbf{0}, \boldsymbol{X}^{\star}\right)$ and use it to construct an approximate solution to the QCQP (12). Note that the specific design of the randomization procedure is problem-dependent. As an illustration, let us consider two representative examples.

\section{Example: Randomization in $B Q P$ or MIMO detection}

For the BQP in (2) or the MIMO detection problem in (11), a typical randomization procedure is as follows.

Box 2. Gaussian Randomization Procedure for BQP

given an SDR solution $\boldsymbol{X}^{\star}$, and a number of randomizations $L$.

for $\ell=1, \ldots, L$

generate $\boldsymbol{\xi}_{\ell} \sim \mathcal{N}\left(\mathbf{0}, \boldsymbol{X}^{\star}\right)$, and construct a QCQP-feasible point

$$
\tilde{\boldsymbol{x}}_{\ell}=\operatorname{sgn}\left(\boldsymbol{\xi}_{\ell}\right) .
$$

end

determine $\ell^{\star}=\arg \min _{\ell=1, \ldots, L} \tilde{\boldsymbol{x}}_{\ell}^{T} \boldsymbol{C} \tilde{\boldsymbol{x}}_{\ell}$.

output $\hat{\boldsymbol{x}}=\tilde{\boldsymbol{x}}_{\ell^{\star}}$ as the approximate QCQP solution.

In Box 2, the problem dependent part lies in (15), where we use rounding to generate feasible points from the random samples $\boldsymbol{\xi}_{\ell}$. Moreover, we repeat the random sampling $L$ times and choose the one that yields the best objective.

In the MIMO detection example in Section III, we have seen that the Gaussian randomization procedure provides quasioptimal bit-error-rate performance; see Fig. 3. Here we give an additional result, plotted in Fig. 5, that shows how the performance improves with the number of randomizations $L$. We see a significant performance gain from $L=1$ to $L=50$. The gain becomes smaller for $L>50$, approaching a limit. This shows that randomization provides an effective approximation for SDR, for sufficient (but not excessive) number of randomizations.

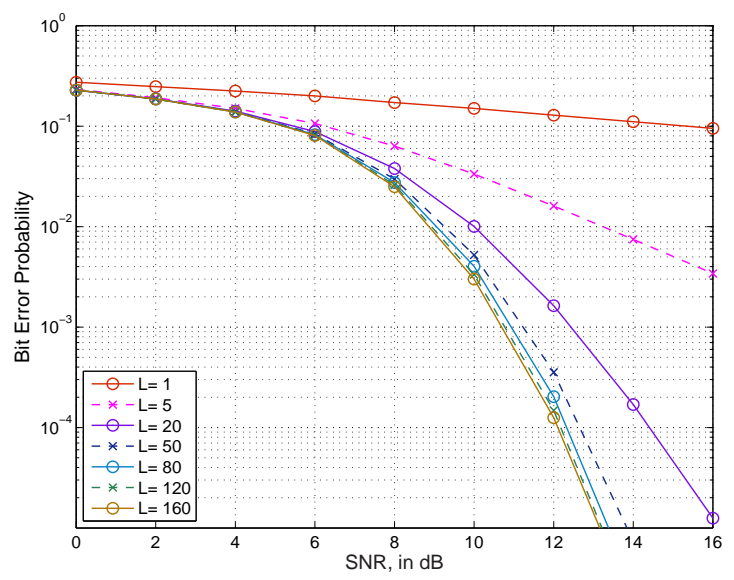

Fig. 5. Performance of various numbers of randomizations in MIMO detection, under the same simulation settings as that in Fig. 3.

\section{Example: Randomization in Problem (3)}

This example aims to geometrically illustrate how randomization behaves. Consider Problem (3), restated here as

$$
\begin{aligned}
& \min _{\boldsymbol{x} \in \mathbb{R}^{n}} \boldsymbol{x}^{T} \boldsymbol{C} \boldsymbol{x} \\
& \text { s.t. } \quad \boldsymbol{x}^{T} \boldsymbol{A}_{i} \boldsymbol{x} \geq 1, \quad i=1, \ldots, m,
\end{aligned}
$$

where $\boldsymbol{C}, \boldsymbol{A}_{1}, \ldots, \boldsymbol{A}_{m} \succeq \mathbf{0}$. Recall that Problem (16) arises in the context of multicast downlink transmit beamforming.

We set up a numerical example where $n=2, m=6$, and then generate many random points $\boldsymbol{\xi} \sim \mathcal{N}\left(\mathbf{0}, \boldsymbol{X}^{\star}\right)$ to see how they distribute in space. An instance of this is shown in Fig. 6. From the distribution of $\boldsymbol{\xi}$ (marked as black '.'), one can see that the covariance matrix $\boldsymbol{X}^{\star}$ is not of rank one, but the density is higher over the direction of the globally optimal QCQP solutions ${ }^{2}$ (marked as green ' $*$ '). Also, note that the random samples $\boldsymbol{\xi}$ are not always feasible for (16), but we can apply a rescaling

$$
\boldsymbol{x}(\boldsymbol{\xi})=\frac{\boldsymbol{\xi}}{\sqrt{\min _{i=1, \ldots, m} \boldsymbol{\xi}^{T} \boldsymbol{A}_{i} \boldsymbol{\xi}}}
$$

to turn them into feasible solutions. We apply the same rescaling to feasible $\boldsymbol{\xi}$, too. The rescaled samples $\boldsymbol{x}(\boldsymbol{\xi})$ are shown as red 'o' in Fig. 6(a). Remarkably, one can see that there is a significant amount of $\boldsymbol{x}(\boldsymbol{\xi})$ that lie close to the optimal QCQP solutions.

A practical randomization procedure for Problem (16) is essentially identical to that presented in Box 2, except that (15) is replaced by (17). Such a procedure has been empirically found to provide promising approximations for the multicast downlink transmit beamforming application and its variations, like the MIMO detection application. Readers are referred to [27], [61] for the results.

\footnotetext{
${ }^{2}$ In this example, the globally optimal QCQP solutions were obtained by a fine grid search on $\mathbb{R}^{2}$. Such an exhaustive search would be prohibitive computationally for general $\mathbb{R}^{n}$.
} 


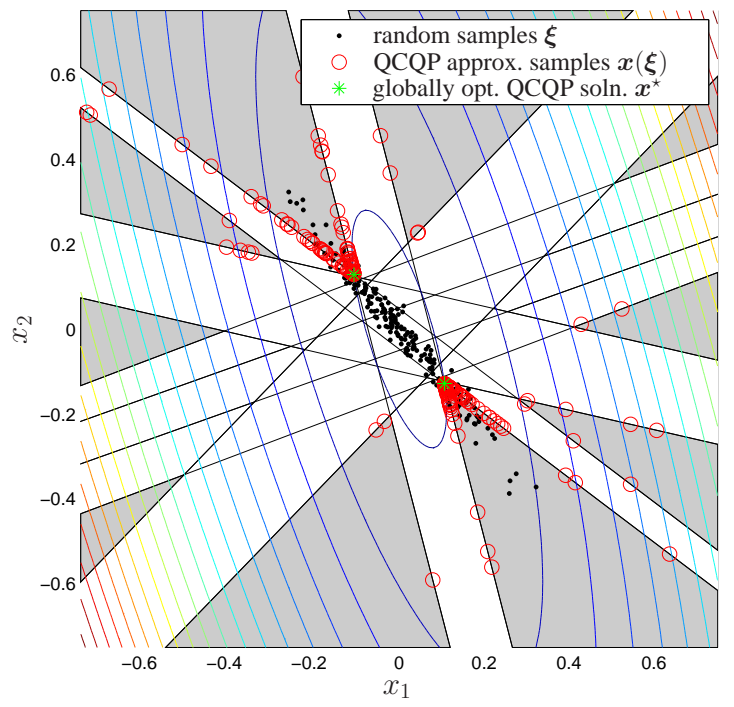

Fig. 6. Illustration of randomizations in $\mathbb{R}^{2}$, for Problem (16). The gray area is the feasible set and colored lines the contour of the objective.

Although we have been using intuitions and illustrations to introduce the randomization approach, the approach is far from being just a heuristic and can in fact yield significant insights into the performance of SDR. Indeed, it was the idea of randomization that opened the gateway to a host of theoretically provable worst-case approximation bounds for SDR. These results have profound implications in applications. For instance, it allows one to get some idea on how well SDR could do if it is to be applied to a new signal processing problem. To give some flavor of these approximation accuracy results, let us first consider Problem (16). Let $v(\boldsymbol{x})=\boldsymbol{x}^{T} \boldsymbol{C} \boldsymbol{x}$ denote the objective function, and let

$$
\begin{aligned}
v_{\mathrm{QP}}=\min _{\boldsymbol{x} \in \mathbb{R}^{n}} & \boldsymbol{x}^{T} \boldsymbol{C} \boldsymbol{x} \\
\text { s.t. } & \boldsymbol{x}^{T} \boldsymbol{A}_{i} \boldsymbol{x} \geq 1, \quad i=1, \ldots, m
\end{aligned}
$$

denote the optimal objective. In [10] (see also [62]), it is shown that with high probability, the objective value $v(\hat{\boldsymbol{x}})$ of a randomized solution $\hat{\boldsymbol{x}}$ will satisfy

$$
v_{\mathrm{QP}} \leq v(\hat{\boldsymbol{x}}) \leq \gamma v_{\mathrm{QP}},
$$

where $\gamma=27 \mathrm{~m}^{2} / \pi$ is the so-called approximation $\mathrm{ra}$ tio. Notice that this ratio accommodates the worst possible problem instance $\left\{\boldsymbol{C}, \boldsymbol{A}_{1}, \ldots, \boldsymbol{A}_{m}\right\}$, and that the practical approximation accuracies can be much better. Fig. 7 gives a numerical evidence, where we show a realization of the actual approximation ratio, $v(\hat{\boldsymbol{x}}) / v_{\mathrm{QP}}$, for the problem instance in Fig. 6. As can be seen from the figure, near optimality is attained for $L \geq 15$. In the same vein, such approximation analysis enables us to know how far the optimal SDR objective value, denoted by $v_{\mathrm{SDR}}=\operatorname{Tr}\left(\boldsymbol{C} \boldsymbol{X}^{\star}\right)$, is from the optimal QCQP value. Indeed, for Problem (16) and its SDR, we can have

$$
v_{\mathrm{SDR}} \leq v_{\mathrm{QP}} \leq \gamma v_{\mathrm{SDR}}
$$

where $\gamma=27 m^{2} / \pi$ is as above.

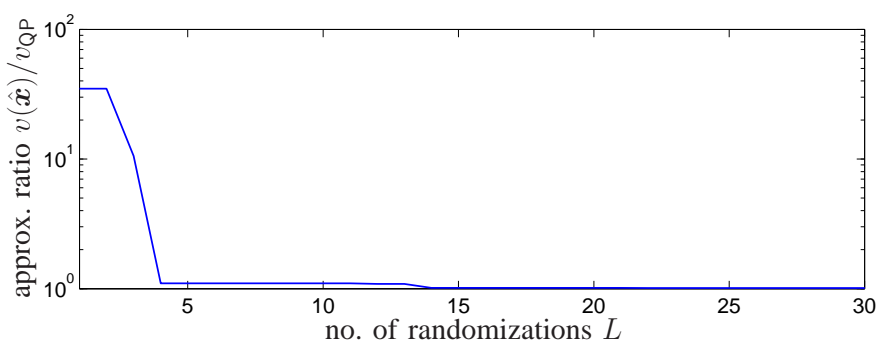

Fig. 7. A realization of the actual approximation accuracy of SDR for the problem instance in Fig. 6.

Now, let us turn to the following class of Boolean quadratic maximization problems $(\mathrm{BQP})$ :

$$
\begin{aligned}
v_{\mathrm{QP}}=\max _{\boldsymbol{x} \in \mathbb{R}^{n}} & \boldsymbol{x}^{T} \boldsymbol{C} \boldsymbol{x} \\
\text { s.t. } & x_{i}^{2}=1, \quad i=1, \ldots, n,
\end{aligned}
$$

with $C \succeq 0$. In the seminal work of Goemans and Williamson [3], it is shown that when $C_{i j} \leq 0$ for all $i \neq j$, one has

$$
\gamma v_{\mathrm{SDR}} \leq v_{\mathrm{QP}} \leq v_{\mathrm{SDR}}
$$

where $\gamma=0.87856$. In addition, if we adopt the randomization procedure in Box 2, then the expected objective value of the randomized solution $\hat{\boldsymbol{x}}$ will satisfy

$$
\gamma v_{\mathrm{QP}} \leq \mathrm{E}\{v(\hat{\boldsymbol{x}})\} \leq v_{\mathrm{QP}}
$$

with the same constant $\gamma$. Although the bounds in (21) apply only to the expected objective value, in practice the randomized solution $\hat{\boldsymbol{x}}$ can often achieve a performance that is well within those bounds.

The analysis of approximation accuracy bounds is a sophisticated subject. Although it is beyond the scope of this paper to elaborate upon the mathematics behind those analyses, we give a summary of some of the major approximation accuracy results in Tables I and II. We refer the interested readers to, e.g., [27], for more technical insights of these results from a signal processing viewpoint.

\section{Extension to More General Cases}

For ease of exposition of the SDR idea, we have only concentrated on the real-valued homogeneous QCQPs in previous sections. Here we illustrate the wide applicability of SDR by showing how the same idea can be used in a number of related problems.

A. Inhomogeneous Problems: Consider a general inhomogeneous QCQP

$$
\begin{aligned}
\min _{\boldsymbol{x} \in \mathbb{R}^{n}} & \boldsymbol{x}^{T} \boldsymbol{C} \boldsymbol{x}+2 \boldsymbol{c}^{T} \boldsymbol{x} \\
\text { s.t. } & \boldsymbol{x}^{T} \boldsymbol{A}_{i} \boldsymbol{x}+2 \boldsymbol{a}_{i}^{T} \boldsymbol{x} \unrhd_{i} b_{i}, \quad i=1, \ldots, m
\end{aligned}
$$

for some appropriate $\boldsymbol{C}, \boldsymbol{c}, \boldsymbol{A}_{i}, \boldsymbol{a}_{i}, b_{i}$. We have already seen in Section III how an inhomogeneous least squares problem can be homogenized. Following the same spirit, we can 
TABLE I

KNOWN APPROXIMATION ACCURACIES OF SDR FOR QUADRATIC MINIMIZATIONS PROBLEMS.

\begin{tabular}{|c|c|c|}
\hline problem & approx. accuracy $\gamma$; see (18)-(19) for def. & references \\
\hline $\begin{aligned} & \min _{\boldsymbol{x} \in \mathbb{C}^{n}} \boldsymbol{x}^{H} \boldsymbol{C} \boldsymbol{x} \\
& \text { s.t. } \boldsymbol{x}^{H} \boldsymbol{A}_{i} \boldsymbol{x} \geq 1, i=1, \ldots, m \\
& \text { where } \boldsymbol{A}_{1}, \ldots, \boldsymbol{A}_{m} \succeq \mathbf{0} .\end{aligned}$ & $\begin{array}{l}\qquad \gamma=8 \mathrm{~m} . \\
\text { If the problem is reduced to the real-valued case, then } \\
\qquad \gamma=\frac{27 \mathrm{~m}^{2}}{\pi} .\end{array}$ & $\begin{array}{l}\text { Luo-Sidiropoulos-Tseng-Zhang [10]; } \\
\text { see also So-Ye-Zhang [62]. } \\
\text { Relevant applications: [61] }\end{array}$ \\
\hline $\begin{array}{l}\text { MIMO Detection } \\
\qquad \min _{\boldsymbol{x} \in \mathbb{R}^{n}} \quad\|\boldsymbol{y}-\boldsymbol{H} \boldsymbol{x}\|_{2}^{2} \\
\text { s.t. } \quad x_{i}^{2}=1, i=1, \ldots, n \\
\text { where } \boldsymbol{y}=\boldsymbol{H} \boldsymbol{s}+\boldsymbol{v} ; \boldsymbol{H} \in \mathbb{C}^{n \times n} \text { has i.i.d. standard } \\
\text { complex Gaussian entries; } s_{i}^{2}=1 \text { for } i=1, \ldots, n \text {; } \\
\text { and } \boldsymbol{v} \in \mathbb{C}^{n} \text { has i.i.d. complex mean zero Gaussian } \\
\text { entries with variance } \sigma^{2} .\end{array}$ & $\begin{array}{l}\text { For } \sigma^{2} \geq 60 n \text { (which corresponds to the low signal-to-noise } \\
\text { ratio (SNR) region), with probability at least } 1-3 \exp (-n / 6), \\
\qquad \gamma \leq \frac{11}{2} . \\
\text { For } \sigma^{2}=\mathcal{O}(1) \text { (which corresponds to the high SNR region), } \\
\text { with probability at least } 1-\exp (-\mathcal{O}(n)), \\
\qquad \gamma=1, \\
\text { i.e. the SDR is tight. }\end{array}$ & $\begin{array}{l}\text { Kisialiou-Luo [59], So-Ye [60]. } \\
\text { Extensions: So-Ye [60]. } \\
\text { Related: Jaldén-Ottersten [58]. } \\
\text { Relevant applications: [17]-[20], [22], } \\
\text { [23] }\end{array}$ \\
\hline
\end{tabular}

homogenize Problem (22) as

$$
\begin{aligned}
\min _{\boldsymbol{x} \in \mathbb{R}^{n}, t \in \mathbb{R}} & {\left[\begin{array}{ll}
\boldsymbol{x}^{T} & t
\end{array}\right]\left[\begin{array}{cc}
\boldsymbol{C} & \boldsymbol{c} \\
\boldsymbol{c}^{T} & 0
\end{array}\right]\left[\begin{array}{l}
\boldsymbol{x} \\
t
\end{array}\right] } \\
\text { s.t. } & t^{2}=1, \\
& {\left[\begin{array}{ll}
\boldsymbol{x}^{T} & t
\end{array}\right]\left[\begin{array}{cc}
\boldsymbol{A}_{i} & \boldsymbol{a}_{i} \\
\boldsymbol{a}_{i}^{T} & 0
\end{array}\right]\left[\begin{array}{l}
\boldsymbol{x} \\
t
\end{array}\right] \unrhd_{i} b_{i}, \quad i=1, \ldots, m, }
\end{aligned}
$$

where both the problem size and the number of constraints increase by one. Hence, SDR can be applied to inhomogeneous QCQPs by operating on their homogenized forms.

Readers are referred to [48], [64] for another interpretation of SDR in the inhomogeneous case.

B. Complex-Valued Problems: Consider a general complexvalued homogeneous QCQP

$$
\begin{aligned}
\min _{\boldsymbol{x} \in \mathbb{C}^{n}} & \boldsymbol{x}^{H} \boldsymbol{C} \boldsymbol{x} \\
\text { s.t. } & \boldsymbol{x}^{H} \boldsymbol{A}_{i} \boldsymbol{x} \unrhd_{i} b_{i}, \quad i=1, \ldots, m,
\end{aligned}
$$

where $\boldsymbol{C}, \boldsymbol{A}_{1}, \ldots, \boldsymbol{A}_{m} \in \mathbb{H}^{n}$, with $\mathbb{H}^{n}$ being the set of all complex $n \times n$ Hermitian matrices. Using the same SDR idea as in the real case, we can derive the following SDR for (23):

$$
\begin{aligned}
\min _{\boldsymbol{X} \in \mathbb{H}^{n}} & \operatorname{Tr}(\boldsymbol{C} \boldsymbol{X}) \\
\text { s.t. } & \operatorname{Tr}\left(\boldsymbol{A}_{i} \boldsymbol{X}\right) \unrhd_{i} b_{i}, \quad i=1, \ldots, m, \\
& \boldsymbol{X} \succeq \mathbf{0},
\end{aligned}
$$

where the only difference is that the problem domain now becomes $\mathbb{H}^{n}$ (in our CVX code insert in Box 1, all you need to do is to change 'symmetric' to 'hermitian'!)

While the SDRs in the real and complex cases are developed using essentially the same technique, it should be noted that the two can be quite different in their approximation accuracies; see, for example, Tables I and II and the literature [27].

The current applications of complex-valued SDR lie in various kinds of beamforming problems [1], [15], [16], [27], [35], [37], [61]. Complex-valued SDR can also be used to handle a $k$-ary quadratic program:

$$
\begin{aligned}
\min _{\boldsymbol{x} \in \mathbb{C}^{n}} & \boldsymbol{x}^{H} \boldsymbol{C} \boldsymbol{x} \\
\text { s.t. } & x_{i} \in\left\{1, e^{j 2 \pi / k}, \ldots, e^{j 2 \pi(k-1) / k}\right\}, i=1, \ldots, n,
\end{aligned}
$$

where $k \geq 2$ is a given integer. Applications of the $k$-ary quadratic program include $M$-ary PSK MIMO detection [20] and coded waveform designs in radar [33]. Problem (25) can be approximated by the following SDR:

$$
\begin{aligned}
\min _{\boldsymbol{X} \in \mathbb{H}^{n}} & \operatorname{Tr}(\boldsymbol{C X}) \\
\text { s.t. } & \boldsymbol{X} \succeq \mathbf{0}, \quad X_{i i}=1, i=1, \ldots, n .
\end{aligned}
$$

Curiously, while the SDR in (26) does not utilize the constellation size $k$, it can yield satisfactory approximations, both practically [20], [33] and theoretically [8], [9].

C. Separable QCQPs: Consider a QCQP of the form

$$
\begin{aligned}
\min _{\boldsymbol{x}_{1}, \ldots, \boldsymbol{x}_{k} \in \mathbb{C}^{n}} & \sum_{i=1}^{k} \boldsymbol{x}_{i}^{H} \boldsymbol{C}_{i} \boldsymbol{x}_{i} \\
\text { s.t. } & \sum_{l=1}^{k} \boldsymbol{x}_{l}^{H} \boldsymbol{A}_{i, l} \boldsymbol{x}_{l} \unrhd_{i} b_{i}, \quad i=1, \ldots, m .
\end{aligned}
$$

Problem (27) is called a separable QCQP. A relevant application for separable QCQPs is the unicast downlink transmit beamforming problem [65]; see [1] in this special issue for the problem description.

Let $\boldsymbol{X}_{i}=\boldsymbol{x}_{i} \boldsymbol{x}_{i}^{T}$ for $i=1, \ldots, k$. By relaxing the rank constraint on each $\boldsymbol{X}_{i}$, we obtain the following SDR of (27):

$$
\begin{aligned}
\min _{\boldsymbol{X}_{1}, \ldots, \boldsymbol{X}_{k} \in \mathbb{H}^{n}} & \sum_{i=1}^{k} \operatorname{Tr}\left(\boldsymbol{C}_{i} \boldsymbol{X}_{i}\right) \\
\text { s.t. } & \sum_{l=1}^{k} \operatorname{Tr}\left(\boldsymbol{A}_{i, l} \boldsymbol{X}_{l}\right) \unrhd_{i} b_{i}, \quad i=1, \ldots, m, \\
& \boldsymbol{X}_{1} \succeq \mathbf{0}, \ldots, \boldsymbol{X}_{k} \succeq \mathbf{0} .
\end{aligned}
$$

\section{Application: Transmit $B_{1}$ Shim in MRI}

At this point readers may have the following concern: since SDR is an approximation method, as an alternative we may also choose to approximate a nonconvex QCQP by an available nonlinear programming method (NPM) (e.g., sequential quadratic programming, available in the MATLAB Optimization Toolbox). Hence, it is natural to ask which method is better. The interesting argument is that they complement each other, instead of competing. Indeed, the quality of NPMs depends on the starting point, and the missing piece 
TABLE II

KNOWN APPROXIMATION ACCURACIES OF SDR FOR QUADRATIC MAXIMIZATION PROBLEMS.

\begin{tabular}{|c|c|c|}
\hline problem & approx. accuracy $\gamma$; see (20)-(21) for def. & references \\
\hline $\begin{array}{l}\text { Boolean QP } \\
\qquad \begin{aligned} \max _{\boldsymbol{x} \in \mathbb{R}^{n}} & \boldsymbol{x}^{T} \boldsymbol{C} \boldsymbol{x} \\
\text { s.t. } & x_{i}^{2}=1, i=1, \ldots, n\end{aligned}\end{array}$ & $\gamma= \begin{cases}0.87856, & \boldsymbol{C} \succeq \mathbf{0}, C_{i j} \leq 0 \forall i \neq j \\
2 / \pi \simeq 0.63661, & \boldsymbol{C} \succeq \mathbf{0} \\
1 \text { (opt.), } & C_{i j} \geq 0, \forall i \neq j\end{cases}$ & $\begin{array}{l}\text { Goemans-Williamson [3], } \\
\text { Nesterov [4], Zhang [7]. } \\
\text { Relevant applications: [24]-[26] }\end{array}$ \\
\hline $\begin{array}{l}\text { Complex } k \text {-ary } \mathrm{QP} \\
\qquad \begin{aligned} \max _{\boldsymbol{x} \in \mathbb{C}^{n}} & \boldsymbol{x}^{H} \boldsymbol{C} \boldsymbol{x} \\
\text { s.t. } & x_{i} \in\left\{1, \omega, \ldots, \omega^{k-1}\right\}, \\
& i=1, \ldots, n\end{aligned} \\
\text { where } \omega=e^{j 2 \pi / k}, \text { and } k>1 \text { is an integer. }\end{array}$ & $\begin{array}{l}\text { For } \boldsymbol{C} \succeq \mathbf{0}, \\
\qquad \gamma=\frac{(k \sin (\pi / k))^{2}}{4 \pi} . \\
\text { e.g., } \gamma=0.7458 \text { for } k=8, \gamma=0.7754 \text { for } k=16 \text {. }\end{array}$ & $\begin{array}{l}\text { Zhang-Huang [8], } \\
\text { So-Zhang-Ye [9]. } \\
\text { Relevant applications: [33] }\end{array}$ \\
\hline 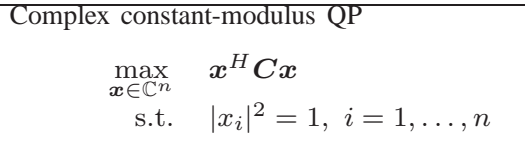 & $\begin{array}{l}\text { For } \boldsymbol{C} \succeq \mathbf{0}, \\
\qquad \gamma=\pi / 4=0.7854 \text {. } \\
\text { Remark: coincide with complex } k \text {-ary QP as } k \rightarrow \infty\end{array}$ & $\begin{array}{l}\text { Zhang-Huang [8], } \\
\text { So-Zhang-Ye [9]. }\end{array}$ \\
\hline 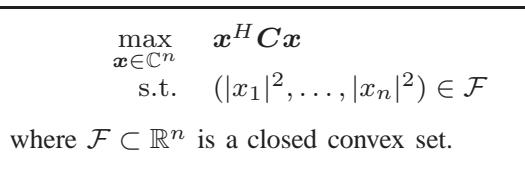 & $\begin{array}{l}\text { The same approx. ratio as in complex constant-modulus QP; } \\
\text { i.e., } \gamma=\pi / 4 \text { for } \boldsymbol{C} \succeq \mathbf{0} \text {. } \\
\text { If the problem is reduced to the real-valued case, then the } \\
\text { approx. ratio results are the same as that in Boolean QP. }\end{array}$ & Ye [5], Zhang [7]. \\
\hline 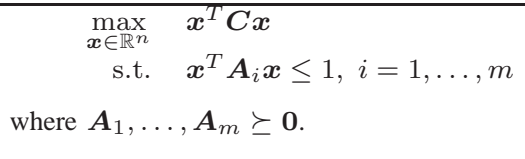 & $\begin{array}{l}\text { For any } \boldsymbol{C} \in \mathbb{S}^{n}, \\
\qquad \gamma=\frac{1}{2 \ln (2 m \mu)} \\
\text { where } \mu=\min \left\{m, \max _{i} \operatorname{rank}\left(\boldsymbol{A}_{i}\right)\right\} .\end{array}$ & $\begin{array}{l}\text { Nemirovski-Roos-Terlaky [6]. } \\
\text { Extensions: Luo-Sidiropoulos-Tseng- } \\
\text { Zhang [10], So-Ye-Zhang [62], and } \\
\text { Zhang-So [63]. }\end{array}$ \\
\hline
\end{tabular}

is generally in securing a reliable (or a 'good enough') starting point. Thus, one can consider a two-stage approach, in which SDR is used to provide a starting point for an NLM. In particular, to SDR, nonlinear programming can provide local refinement of the solution, while to NLMs SDR can be used to provide a good starting point. This two-stage approach has not only been proven to be viable in practice, but is also promising in performance [28], [37].

In this example we demonstrate the effectiveness of the two-stage approach. The application involved is transmit $B_{1}$ shimming in magnetic resonance imaging (MRI) [37]. An illustration is shown in Fig. 8 to help us explain the problem. A magnetic field, specifically a $B_{1}$ field is generated by an array of transmit RF coils. The ideal situation would be that the $B_{1}$ field is spatially uniform across the load (like, a human head). Unfortunately, this is usually not the case. The complex interactions between the magnetic field and the loaded tissues often result in strong inhomogeneity (or spatial non-uniformity) across the load. The goal of transmit $B_{1}$ shimming is to design the transmit amplitudes and phases of the RF coils such that the resultant $B_{1}$ map (or the MR image) is as uniform as possible.

The transmit $B_{1}$ shimming problem is mathematically formulated as follows. Let $\boldsymbol{x} \in \mathbb{C}^{n}$ be the transmit vector of the $\mathrm{RF}$ coil array, where $n$ is the number of RF coils and $x_{i}$ is a complex variable characterizing the transmit amplitude and phase of the $i$ th RF coil. Denote by $\boldsymbol{a}_{i} \in \mathbb{C}^{n}, i=1, \ldots, m$,

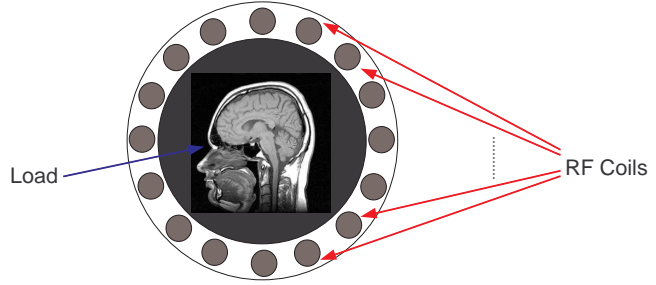

Fig. 8. An MRI illustration.

the field response from the array to the $i$ th pixel; that is to say, the $i$ th pixel receives a $B_{1}$ field of magnitude $\left|\boldsymbol{a}_{i}^{T} \boldsymbol{x}\right|$. Our problem then is to minimize the worst-case field magnitude difference

$$
\begin{aligned}
\min _{\boldsymbol{x} \in \mathbb{C}^{n}} & \left.\max _{i=1, \ldots, m}|| \boldsymbol{a}_{i}^{T} \boldsymbol{x}\right|^{2}-b^{2} \mid \\
\text { s.t. } & \boldsymbol{x}^{H} \boldsymbol{G} \boldsymbol{x} \leq \rho .
\end{aligned}
$$

Here, $m$ is the total number of pixels, $b>0$ is the desired pixel value (which is uniform over all pixels), $\boldsymbol{x}^{H} \boldsymbol{G} \boldsymbol{x}$ represents the average specific absorption rate (SAR), in which $G$ is composed of the complex-valued $E$ field coefficients and of the tissue conductivity and mass density, and $\rho$ is a prespecified SAR limit.

Let us consider an SDR of Problem (29), which, by following the SDR principles mentioned in previous sections, is 
given by

$$
\begin{aligned}
\min _{\boldsymbol{X} \in \mathbb{H}^{n}} & \max _{i=1, \ldots, m}\left|\operatorname{Tr}\left(\boldsymbol{a}^{*} \boldsymbol{a}_{i}^{T} \boldsymbol{X}\right)-b^{2}\right| \\
\text { s.t. } & \boldsymbol{X} \succeq \mathbf{0}, \operatorname{Tr}(\boldsymbol{G} \boldsymbol{X}) \leq \rho .
\end{aligned}
$$

Note that the SDR problem in (30) can be reformulated as an SDP:

$$
\begin{array}{rl}
\min _{t \in \mathbb{R},} \boldsymbol{X} \in \mathbb{H}^{n} & t \\
\text { s.t. } & -t \leq \operatorname{Tr}\left(\boldsymbol{a}^{*} \boldsymbol{a}_{i}^{T} \boldsymbol{X}\right)-b^{2} \leq t, i=1, \ldots, m, \\
& \boldsymbol{X} \succeq \mathbf{0}, \operatorname{Tr}(\boldsymbol{G} \boldsymbol{X}) \leq \rho .
\end{array}
$$

A randomization procedure reminiscent of that given in Box 2 can be used to generate an approximate solution to the original problem in (29); see [37] for the algorithm description.

A simulation result for transmit $B_{1}$ shimming is shown in Fig. 9. We employ a 16-element RF strip line coil array, operating at 7 Tesla and loaded with a human head model. Fig. 9(a) shows a $B_{1}$ map obtained by a simple, non-optimized transmit weight $\boldsymbol{x}=\left[1, e^{2 \pi / 16}, \ldots, e^{30 \pi / 16}\right]^{T}$. From that figure and its respective objective value (provided below the figure), we can see that the resultant $B_{1}$ map is not uniform enough. Figs. 9(d) and (e) show the results for SDR randomized solutions, where the number of randomizations is $L=200$. Randomization would lead to variations in different runs or realizations. Due to space limit, we only display two realizations in Figs. 9(d) and (e). One can observe that there are some differences with the $B_{1}$ maps of the two realizations, but their objective values are quite similar. The randomized SDR solutions also show improvements in uniformity when compared to the non-optimized transmit weight in Fig. 9(a). Now, let us consider the two-stage approach mentioned in the beginning of this section. The results are shown in Figs. 9(f) and $(\mathrm{g})$. We can see further improvements with the resultant $B_{1}$ maps and objective values. This shows that SDR can provide reliable initializations to NPMs.

One may also be interested in seeing how an NPM perform without the aid of SDR. To do this comparison, we randomly generate a starting point for the NPM by an i.i.d. Gaussian distribution. However, for fairness of comparison to SDR, we generate $L$ i.i.d. Gaussian random points (the same $L$ as in randomization in SDR) and set the starting point to be the one that yields the best objective. Two $B_{1}$ map realizations of such randomly initialized NPM are shown in Figs. 9(b) and (c). We can see that the performance shows significant variations from one realization to another (it could be good, and it could be bad), making the final solution fidelity difficult to say. In [37], some Monte Carlo simulations are provided to further support our observations here.

\section{RANK REDUCTION IN SDP}

As the readers may have noticed by now, one of the recurring themes in the SDR methodology is the following. First, one formulates a given hard optimization problem as a rank-constrained SDP. Then, one removes the rank constraint to obtain an SDP. This is vividly illustrated as we pass from the QCQP (4) to the equivalent rank-constrained SDP (5), and finally to the SDR (6). Now, if the algorithm we use to solve the SDP returns a solution whose rank satisfies the original rank constraint, then that solution will also be optimal for the original problem. As the applications we consider typically require that the solution matrix has low rank (e.g., the solution matrix in Problem (5) must have rank one), it is natural to ask whether standard interior-point algorithms for solving SDPs will return a low rank solution or not. Unfortunately, the answer is no in general. Specifically, it has been shown [66] that standard interior-point algorithms for solving SDPs will always return a solution whose rank is maximal among all optimal solutions. Thus, either the problem at hand possesses some very special structure, or we have to be somewhat lucky in order to obtain a low rank SDP solution. On the other hand, not all is lost. It turns out that if an SDP with an $n \times n$ matrix variable and $m$ linear constraints is feasible, then there always exists a solution whose rank is bounded above by $\mathcal{O}(\sqrt{m})$. Specifically, Shapiro [67], and later Barvinok [68] and Pataki [69] independently showed that if the SDP (6) is feasible, then there exists a solution $X^{\star}$ to (6) such that

$$
\frac{\operatorname{rank}\left(\boldsymbol{X}^{\star}\right)\left(\operatorname{rank}\left(\boldsymbol{X}^{\star}\right)+1\right)}{2} \leq m,
$$

or equivalently, $\operatorname{rank}\left(\boldsymbol{X}^{\star}\right) \leq\lfloor(\sqrt{8 m+1}-1) / 2\rfloor$. Moreover, such a solution can be found efficiently [69]. The ShapiroBarvinok-Pataki (SBP) result has many interesting consequences. For instance, when $m \leq 2$, we have $\operatorname{rank}\left(\boldsymbol{X}^{\star}\right) \leq 1$ whenever (6) is feasible. This implies that the SDP (6) is equivalent to the rank-constrained SDP (5). In particular, we can obtain an optimal solution to the seemingly difficult Problem (4) simply by solving an SDP.

As it turns out, a similar SDR rank result holds for the complex-valued homogeneous QCQP (23) and the separable QCQP (27). Specifically, Huang and Palomar [16] showed that if the SDR (24) of the complex-valued homogeneous QCQP (23) is feasible, then there exists a solution $\boldsymbol{X}^{\star}$ to (24) such that $\operatorname{rank}\left(\boldsymbol{X}^{\star}\right) \leq \sqrt{m}$. On the other hand, consider the SDR (28) of the complex-valued separable QCQP (27). Suppose that it is feasible. Then, as shown in [16], there exists a solution $\left\{\boldsymbol{X}_{i}^{\star}\right\}_{i=1}^{k}$ to (28) whose ranks satisfy

$$
\sum_{i=1}^{k} \operatorname{rank}\left(\boldsymbol{X}_{i}^{\star}\right)^{2} \leq m .
$$

In the case of a real-valued separable QCQP, the rank condition is given by

$$
\sum_{i=1}^{k} \frac{\operatorname{rank}\left(\boldsymbol{X}_{i}^{\star}\right)\left(\operatorname{rank}\left(\boldsymbol{X}_{i}^{\star}\right)+1\right)}{2} \leq m .
$$

To summarize:

For a real-valued (resp. complex-valued) homogeneous QCQP with 2 (resp. 3) constraints or less, SDR is not just a relaxation. It is tight, i.e., solving the SDR is equivalent to solving the original QCQP.

For a homogeneous separable QCQP (27), suppose that none of the solution $\left\{\boldsymbol{X}_{i}^{\star}\right\}_{i=1}^{k}$ to the SDR (28) satisfies $\boldsymbol{X}_{i}^{\star}=\mathbf{0}$ for some $i$. Then, the SDR is tight if $m \leq k+2$ in the complex case; and if $m \leq k+1$ in the real case. 


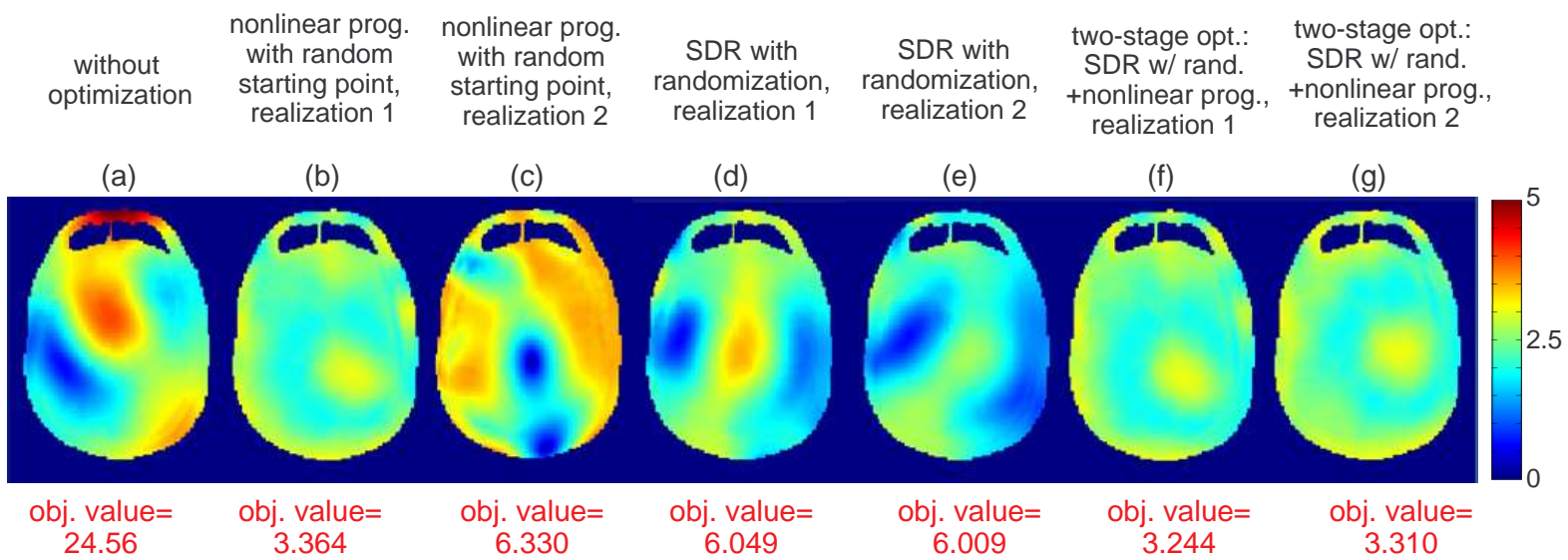

Fig. 9. $B_{1}$ maps of various optimization methods.

An important application of the above result is in establishing the tightness of certain SDR for the unicast downlink transmit beamforming problem; see [1], [16], [27] for further discussions.

Before we proceed further, several remarks are in order.

1) The SBP result is concerned with the existence of low rank solutions to an SDP, and we derive the tightness of various SDRs as corollaries (by specializing the SBP result to the rank one case). However, there are other, more direct, approaches for proving tightness of SDRs of various QCQPs; see, e.g., [12]-[14], [70], [71]. Most of these approaches rely on so-called rank-one decomposition theorems, which allow one to extract an optimal QCQP solution from the SDR solution, provided that the number of constraints in the QCQP is not too large-say, at most 3 for the complex-valued homogeneous QCQP. Recently, Ai et al. [71] have proven another rank-one decomposition theorem and used it to show that the SDRs of a large class of complex-valued homogeneous QCQPs with 4 constraints are in fact tight. The interested readers may find the MATLAB implementations of the algorithms described in [71] at http://www.se.cuhk . edu.hk/ ywhuang/dcmp/paper.html. We note that the aforementioned tightness results have already found many applications in signal processing and communications; see, e.g., [32], [33], [40], [41], [71]-[75].

2) It is known [68] that the rank bound in (32) cannot be improved in general. Specifically, there exist SDPs with $m$ constraints in which every matrix that satisfies all the constraints must have rank of order at least $\sqrt{m}$. However, if one allows the linear constraints in a given SDP to be satisfied only approximately, then it is possible to find a solution matrix whose rank is much smaller than $\mathcal{O}(\sqrt{m})$. We refer the readers to [62] for details.

3) The results mentioned in this section merely provide sufficient conditions for SDR tightness. As such, there are cases in which SDR tightness can be attained under different conditions. For example, if each $\boldsymbol{A}_{i}$ follows the structure

$$
\boldsymbol{A}_{i}=\boldsymbol{a}_{i} \boldsymbol{a}_{i}^{H}, \quad \boldsymbol{a}_{i}=\left[1, e^{j \phi_{i}}, \ldots, e^{j(n-1) \phi_{i}}\right]^{T}
$$

for some angle $\phi_{i} \in[0,2 \pi)$, then a rank-one solution exists for SDR for any $m$ [15]. Another example is in MIMO detection, where SDR tightness can be shown to occur with high probability [57], [59], [60], [76].

\section{Application: SENSOR Network LOCALIZATiON}

Let us now consider another practical problem to which the SDR technique can be applied, namely, the sensor network localization (SNL) problem. Although the SNL problem is computationally intractable, it can be relaxed to an SDP. Moreover, simulation results showed that it can produce high quality solutions. Before we delve into the details, let us first briefly describe and motivate the SNL problem.

In recent years, the deployment of large-scale wireless sensor networks has become increasingly common. These networks are often used to collect location-dependent data, such as motion at various points of a monitored area, temperature at various locations of a habitat, etc. In most applications, however, the sensors are deployed in an ad-hoc fashion. Moreover, it is often impractical or infeasible to equip every sensor with a location device (such as GPS). Thus, the actual locations of individual sensors may not be known, and we need to deduce them from some other information. One common approach is to use the so-called communication graph of the sensors. Specifically, consider a graph in which the nodes represent sensors, and an edge between two nodes indicates that the corresponding sensors can communicate with each other. We assume that the distance between two sensors can be measured whenever they can communicate with each other ${ }^{3}$. To add some flexibility to the model, we allow for the possibility that the locations of some of the sensors are given. These sensors will be referred to as anchors in the sequel.

Under the above setting, our goal is to determine the coordinates of the sensors in, say $\mathbb{R}^{2}$, so that the distances induced

\footnotetext{
${ }^{3}$ This can be achieved using, e.g., the arrival time or difference in arrival time of the signal, the received signal strength, or angle of arrival measurements (see, e.g., [77], [78] and references therein).
} 
by those coordinates match the measured distances. Formally, let $V_{s}=\{1, \ldots, n\}$ and $V_{a}=\{n+1, \ldots, n+m\}$ be the sets of sensors and anchors, respectively. Let $E_{s s}$ and $E_{s a}$ be the sets of sensor-sensor and sensor-anchor edges, respectively. To fix ideas and keep our exposition simple, suppose for now that the measured distances $\left\{d_{i k}:(i, k) \in E_{s s}\right\}$ and $\left\{\bar{d}_{i k}:(i, k) \in E_{s a}\right\}$ are noise-free. Then, the SNL problem becomes that of finding $\boldsymbol{x}_{1}, \ldots, \boldsymbol{x}_{n} \in \mathbb{R}^{2}$ such that

$$
\begin{array}{ll}
\left\|\boldsymbol{x}_{i}-\boldsymbol{x}_{k}\right\|^{2}=d_{i k}^{2}, & (i, k) \in E_{s s}, \\
\left\|\boldsymbol{a}_{i}-\boldsymbol{x}_{k}\right\|^{2}=\bar{d}_{i k}^{2}, & (i, k) \in E_{s a} .
\end{array}
$$

In general, Problem (33) is difficult to solve, as the quadratic constraints in it are nonconvex. Indeed, the problem of determining the feasibility of (33) is NP-hard [79]. However, one can derive a computationally efficient SDR of Problem (33) as follows. First, observe that

$$
\left\|\boldsymbol{x}_{i}-\boldsymbol{x}_{k}\right\|^{2}=\boldsymbol{x}_{i}^{T} \boldsymbol{x}_{i}-2 \boldsymbol{x}_{i}^{T} \boldsymbol{x}_{k}+\boldsymbol{x}_{k}^{T} \boldsymbol{x}_{k} .
$$

In particular, we see that $\left\|\boldsymbol{x}_{i}-\boldsymbol{x}_{k}\right\|^{2}$ is linear in the inner products $\boldsymbol{x}_{i}^{T} \boldsymbol{x}_{i}, \boldsymbol{x}_{i}^{T} \boldsymbol{x}_{k}$ and $\boldsymbol{x}_{k}^{T} \boldsymbol{x}_{k}$. Hence, we may write

$$
\left\|\boldsymbol{x}_{i}-\boldsymbol{x}_{k}\right\|^{2}=\left(\boldsymbol{e}_{i}-\boldsymbol{e}_{k}\right)^{T} \boldsymbol{X}^{T} \boldsymbol{X}\left(\boldsymbol{e}_{i}-\boldsymbol{e}_{k}\right)=\operatorname{Tr}\left(\boldsymbol{E}_{i k} \boldsymbol{X}^{T} \boldsymbol{X}\right),
$$

where $\boldsymbol{e}_{i} \in \mathbb{R}^{n}$ is the $i$-th unit vector, $\boldsymbol{E}_{i k}=\left(\boldsymbol{e}_{i}-\boldsymbol{e}_{k}\right)\left(\boldsymbol{e}_{i}-\right.$ $\left.\boldsymbol{e}_{k}\right)^{T} \in \mathbb{S}^{n}$, and $\boldsymbol{X}$ is a $2 \times n$ matrix whose $i$-th column is $\boldsymbol{x}_{i}$. In a similar fashion, we have

$$
\left\|\boldsymbol{a}_{i}-\boldsymbol{x}_{k}\right\|^{2}=\boldsymbol{a}_{i}^{T} \boldsymbol{a}_{i}-2 \boldsymbol{a}_{i}^{T} \boldsymbol{x}_{k}+\boldsymbol{x}_{k}^{T} \boldsymbol{x}_{k} .
$$

Although the term $\boldsymbol{a}_{i}^{T} \boldsymbol{x}_{k}$ is linear only in $\boldsymbol{x}_{k}$, we may homogenize it and write

$$
\begin{aligned}
\left\|\boldsymbol{a}_{i}-\boldsymbol{x}_{k}\right\|^{2} & =\left[\begin{array}{ll}
\boldsymbol{a}_{i}^{T} & \boldsymbol{e}_{k}^{T}
\end{array}\right]\left[\begin{array}{cc}
\boldsymbol{I}_{2} & \boldsymbol{X} \\
\boldsymbol{X}^{T} & \boldsymbol{X}^{T} \boldsymbol{X}
\end{array}\right]\left[\begin{array}{c}
\boldsymbol{a}_{i} \\
\boldsymbol{e}_{k}
\end{array}\right] \\
& =\operatorname{Tr}\left(\overline{\boldsymbol{M}}_{i k} \boldsymbol{Z}\right)
\end{aligned}
$$

where

$$
\overline{\boldsymbol{M}}_{i k}=\left[\begin{array}{c}
\boldsymbol{a}_{i} \\
\boldsymbol{e}_{k}
\end{array}\right]\left[\begin{array}{ll}
\boldsymbol{a}_{i}^{T} & \boldsymbol{e}_{k}^{T}
\end{array}\right]
$$

and

$$
\boldsymbol{Z}=\left[\begin{array}{cc}
\boldsymbol{I}_{2} & \boldsymbol{X} \\
\boldsymbol{X}^{T} & \boldsymbol{X}^{T} \boldsymbol{X}
\end{array}\right]=\left[\begin{array}{c}
\boldsymbol{I}_{2} \\
\boldsymbol{X}^{T}
\end{array}\right]\left[\begin{array}{cc}
\boldsymbol{I}_{2} & \boldsymbol{X}
\end{array}\right]
$$

Now, observe that $\boldsymbol{Z} \in \mathbb{S}^{n+2}$ as given in (34) is a rank 2 positive semidefinite matrix whose upper left $2 \times 2$ block is constrained to be an identity matrix. The latter can be expressed as three linear constraints (i.e., linear in the entries of $\boldsymbol{Z}$ ). Moreover, using the Schur complement, it is not hard to show that any rank 2 positive semidefinite matrix $\boldsymbol{Z} \in \mathbb{S}^{n+2}$ whose upper left $2 \times 2$ block is an identity matrix must have the form given in (34) for some $\boldsymbol{X} \in \mathbb{R}^{2 \times n}$. Thus, upon letting

$$
\boldsymbol{M}_{i k}=\left[\begin{array}{cc}
\mathbf{0} & \mathbf{0} \\
\mathbf{0} & \boldsymbol{E}_{i k}
\end{array}\right],
$$

we see that Problem (33) is equivalent to the following rank constrained SDP:

$$
\begin{array}{cl}
\text { find } & \boldsymbol{Z} \\
\text { s.t. } & \operatorname{Tr}\left(\boldsymbol{M}_{i k} \boldsymbol{Z}\right)=d_{i k}^{2}, \quad(i, k) \in E_{s s}, \\
& \operatorname{Tr}\left(\overline{\boldsymbol{M}}_{i k} \boldsymbol{Z}\right)=\bar{d}_{i k}^{2}, \quad(i, k) \in E_{s a}, \\
& \boldsymbol{Z}_{1: 2,1: 2}=\boldsymbol{I}_{2}, \\
& \boldsymbol{Z} \succeq \mathbf{0}, \quad \operatorname{rank}(\boldsymbol{Z})=2 .
\end{array}
$$

In particular, by dropping the rank constraint from (35), we obtain an SDR of Problem (33).

Now, if we solve the SDR of Problem (33) and obtain a rank $r$ solution $Z$, then we can extract from it a set of $r$-dimensional coordinates for the sensors such that those coordinates satisfy the distance constraints [30]. In fact, if the solution $Z$ is of rank 2 , then we can extract the two-dimensional coordinates of the sensors directly from the $\boldsymbol{X}$ portion of the matrix $Z$ (see (34)). For other interesting theoretical properties of the above SDR, we refer the readers to [28], [30], [80].

So far our discussion has focused on the case where the measured distances are noise-free. However, in practice, the measured distances are usually corrupted by noise (say, by an additive Gaussian noise). In this case, we are interested in finding a maximum likelihood estimate (MLE) of the sensors' coordinates. Although the MLE problem is difficult to solve in general, one can derive an SDR of it using techniques similar to those introduced in this section. We refer the readers to [28], [31] for details.

To demonstrate the power of the SDR approach, we applied it to a randomly generated network of 45 sensors and 5 anchors over the unit square $[-0.5,0.5]^{2}$. The connectivity of the network is determined by the so-called unit disk graph model. Specifically, we assume that a pair of devices can communicate with each other if the distance between them is at most 0.3. Furthermore, we assume that the measured distances are corrupted by a Gaussian noise with small variance, say 0.01 .

In Fig. 10(a) we show the positions of the sensors as computed by the SDP, as well as the trajectories of a gradient search procedure after initializing it with the SDP solution. We use circles ' $O$ ' to denote the true positions of the sensors and diamonds ' $\checkmark$ ' to denote the positions of the anchors. The initial positions of the sensors as computed by the SDP are denoted by stars ' $*$ ', and the tail end of a trajectory gives the computed position of a sensor after 50 iterations of the gradient search procedure. As can be seen from the figure, the final computed positions of the sensors are very close to the true positions. For the purpose of comparison and to demonstrate the high quality of the SDP solution, we show in Fig. 10(b) the trajectories of the gradient search procedure when it is initialized by a random starting point. As can be seen from the figure, even after 50 iterations, the computed positions of the sensors are still nowhere close to the true positions.

Before we leave this section, we should mention that the SDR technique can also be applied to the source localization problem (see, e.g., [77], [78]), which is well-studied in the signal processing community and may be considered as a special case of the sensor network localization problem. In that problem, one is given noisy distance measurements from one sensor to a number of anchors, and the goal is to determine the MLE of the sensor position. For various SDR-based approaches to this problem, we refer the readers to [31], [38], [39].

\section{Conclusion And Discussion}

In this paper we have provided a general, comprehensive coverage of the SDR technique, from its practical deployments 


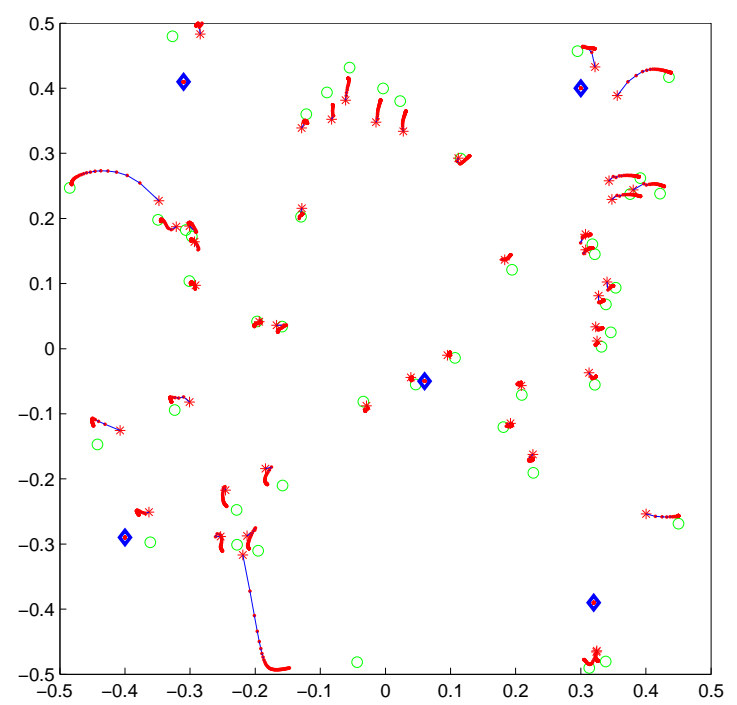

(a) Gradient search initialized by SDP solution

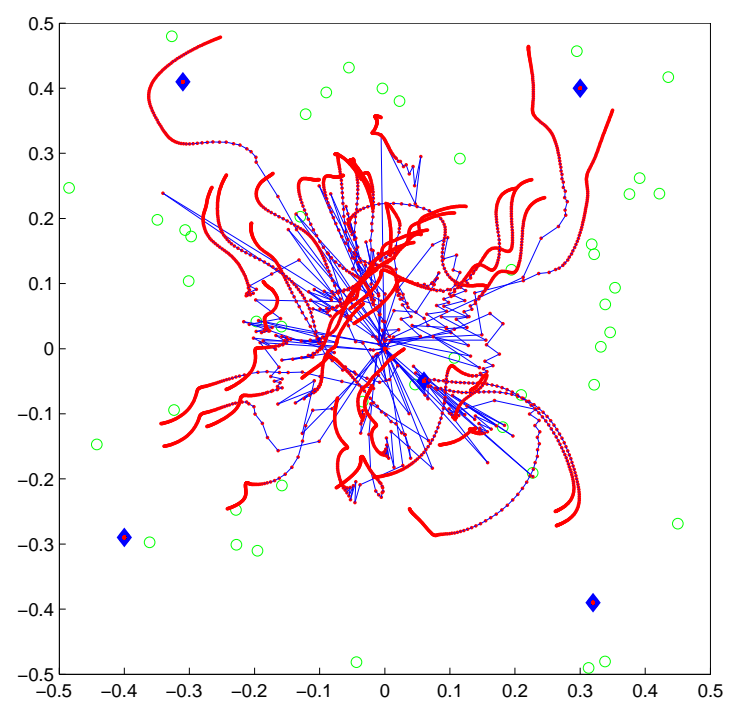

(b) Gradient search initialized by a random starting point

Fig. 10. Refinement through a gradient search method for a 50 node network

and scope of applicability to key theoretical results. We have also showcased several representative applications, namely MIMO detection, $B_{1}$ shimming in MRI and sensor network localization. Another important application, namely downlink transmit beamforming, is described in the article [1] in this special issue. Due to space limit, we are unable to cover many other beautiful applications of the SDR technique, although we have done our best to illustrate the key intuitive ideas that resulted in those applications. We hope that this introductory paper will serve as a good starting point for readers who would like to apply the SDR technique to their applications, and to locate specific references either in applications or theory.

\section{ACKnOWledgments}

This work is supported in part by Hong Kong Research Grants Council (RGC) General Research Funds (GRFs), Project Numbers CUHK415908, CUHK416908, and CUHK419208; by the Army Research Office, Grant Number W911NF-09-1-0279; and by the National Science Foundation, Grant Number CMMI-0726336.

\section{REFERENCES}

[1] A. B. Gershman, N. D. Sidiropoulos, S. Shahbazpanahi, M. Bengtsson, and B. Ottersten, "Convex optimization-based beamforming: From receive to transmit and network designs," IEEE Signal Process. Mag., Special Issue on Convex Optim. for Signal Process., 2010.

[2] L. Lovász, "On the Shannon capacity of a graph," IEEE Trans. Inform. Theory, vol. IT-25, no. 1, pp. 1-7, 1979.

[3] M. X. Goemans and D. P. Williamson, "Improved approximation algorithms for maximum cut and satisfiability problem using semi-definite programming," J. ACM, vol. 42, pp. 1115-1145, 1995.

[4] Yu. E. Nesterov, "Semidefinite relaxation and nonconvex quadratic optimization," Optim. Meth. Softw., vol. 9, pp. 140-160, 1998.

[5] Y. Ye, "Approximating quadratic programming with bound and quadratic constraints," Math. Program., vol. 84, no. 2, pp. 219-226, 1999.

[6] A. Nemirovski, C. Roos, and T. Terlaky, "On maximization of quadratic form over intersection of ellipsoids with common center," Math. Program., vol. 86, pp. 463-473, 1999.

[7] S. Zhang, "Quadratic maximization and semidefinite relaxation," Math. Program., vol. 87, pp. 453-465, 2000.
[8] S. Zhang and Y. Huang, "Complex quadratic optimization and semidefinite programming," SIAM J. Optim., vol. 3, pp. 871-890, 2006.

[9] A. M.-C. So, J. Zhang, and Y. Ye, "On approximating complex quadratic optimization problems via semidefinite programming relaxations," Math. Program., vol. 110, no. 1, pp. 93-110, 2007.

[10] Z.-Q. Luo, N. D. Sidiropoulos, P. Tseng, and S. Zhang, "Approximation bounds for quadratic optimization with homogeneous quadratic constraints," SIAM J. Optim., vol. 18, no. 1, pp. 1-28, Feb. 2007.

[11] S. He, Z.-Q. Luo, J. Nie, and S. Zhang, "Semidefinite relaxation bounds for indefinite homogeneous quadratic optimization," SIAM J. Optim., vol. 19, no. 2, pp. 503-523, 2008.

[12] Y. Ye and S. Zhang, "New results on quadratic minimization," SIAM J. Optim., vol. 14, no. 1, pp. 245-267, 2003.

[13] A. Beck and Y. C. Eldar, "Strong duality in nonconvex quadratic optimization with two quadratic constraints," SIAM J. Optim., vol. 17, no. 3, pp. 844-860, 2003.

[14] Y. Huang and S. Zhang, "Complex matrix decomposition and quadratic programming," Math. Oper. Res., vol. 32, no. 3, pp. 758-768, Aug. 2007.

[15] E. Karipidis, N. D. Sidiropoulos, and Z.-Q. Luo, "Far-field multicast beamforming for uniform linear antenna arrays," IEEE Trans. Signal Process., vol. 55, no. 10, pp. 4916-4927, 2007.

[16] Y. Huang and D. P. Palomar, "Rank-constrained separable semidefinite programming with applications to optimal beamforming," to appear in IEEE Trans. Signal Process., 2009.

[17] P. Tan and L. Rasmussen, "The application of semidefinite programming for detection in CDMA," IEEE J. Select. Areas Commun., vol. 19, no. 8, pp. 1442-1449, Aug. 2001.

[18] W.-K. Ma, T. N. Davidson, K. M. Wong, Z.-Q. Luo, and P. C. Ching, "Quasi-maximum-likelihood multiuser detection using semi-definite relaxation with application to synchronous CDMA," IEEE Trans. Signal Process., vol. 50, no. 4, pp. 912-922, Apr. 2002.

[19] B. Steingrimsson, Z.-Q. Luo, and K. M. Wong, "Soft quasi-maximumlikelihood detection for multiple-antenna wireless channels," IEEE Trans. Signal Process., vol. 51, no. 11, pp. 2710-2719, 2003.

[20] W.-K. Ma, P. C. Ching, and Z. Ding, "Semidefinite relaxation based multiuser detection for M-ary PSK multiuser systems," IEEE Trans. Signal Process., vol. 52, no. 10, pp. 2862-2872, 2004.

[21] M. Kisialiou, X. Luo, and Z.-Q. Luo, "Efficient implementation of quasimaximum-likelihood detection nased on semidefinite relaxation," IEEE Trans. Signal Process., vol. 57, no. 12, pp. 4811-4822, Dec. 2009.

[22] A. Mobasher, M. Taherzadeh, R. Sotirov, and A. K. Khandani, "A near-maximum-likelihood decoding algorithm for MIMO systems based on semi-definite programming," IEEE Trans. Inform. Theory, vol. 53, no. 11 , pp. 3869-3886, 2007.

[23] W.-K. Ma, C.-C. Su, J. Jaldén, T.-H. Chang, and C.-Y. Chi, "The equivalence of semidefinite relaxation MIMO detectors for higher-order QAM," IEEE J. Selected Topics Signal Process., vol. 3, no. 6, pp. 10381052, Dec 2009. 
[24] Q. Li, E.-W. Bai, and Y. Ye, "Blind channel equalization and $\epsilon-$ approximation algorithms," IEEE Trans. Signal Process., vol. 49, no. 1, pp. 2823-2831, Nov. 2001.

[25] Q. Li, E.-W. Bai, and Z. Ding, "Blind source separation of signals with known alphabets using $\epsilon$-approximation algorithms," IEEE Trans. Signal Process., vol. 51, no. 1, pp. 1-10, Jan. 2003.

[26] W.-K. Ma, B.-N. Vo, T. N. Davidson, and P. C. Ching, "Blind ML detection of orthogonal space-time block codes: Efficient high-performance implementations," IEEE Trans. Signal Process., vol. 54, no. 2, pp. 738751, Feb. 2006.

[27] Z.-Q. Luo and T.-H. Chang, "SDP relaxation of homogeneous quadratic optimization: Approximation bounds and applications," in Convex Optimization in Signal Processing and Communications, Chapter 4, D. P. Palomar and Y. C. Eldar, Ed., Cambridge University Press, 2010.

[28] P. Biswas, T.-C. Lian, T.-C. Wang, and Y. Ye, "Semidefinite programming based algorithms for sensor network localization," ACM Trans. Sensor Networks, vol. 2, no. 2, pp. 188-220, 2006.

[29] P. Biswas, T.-C. Liang, K.-C. Toh, Y. Ye, and T.-C. Wang, "Semidefinite programming approaches for sensor network localization with noisy distance measurements," IEEE Trans. Autom. Sci. Eng., vol. 3, no. 4, pp. 360-371, Oct. 2006.

[30] A. M.-C. So and Y. Ye, "Theory of semidefinite programming for sensor network localization," Math. Program., Series B, vol. 109, no. 2-3, pp. 367-384, 2007.

[31] K. W. K. Lui, W.-K. Ma, H. C. So, and F. K. W. Chan, "Semidefinite programming algorithms for sensor network node localization with uncertainties in anchor positions and/or propagation speed," IEEE Trans. Signal Process., vol. 57, no. 2, pp. 752-763, 2009.

[32] A. De Maio, S. De Nicola, Y. Huang, S. Zhang, and A. Farina, "Code design to optimize radar detection performance under accuracy and similarity constraints," IEEE Trans. Signal Process., vol. 56, no. 11, pp. 5618-5629, Nov. 2008.

[33] A. De Maio, S. De Nicola, Y. Huang, Z.-Q. Luo, and S. Zhang, "Design of phase codes for radar performance optimization with a similarity constraint," IEEE Trans. Signal Process., vol. 57, no. 2, pp. 610-621, Feb. 2009.

[34] J.-J. Xiao, Z.-Q. Luo, and M. Jiang, "Two-dimensional phase unwrapping using semidefinite relaxation," in Proc. IEEE Int. Conf. Acoustics, Speech, and Signal Process., Taipei, Taiwan, Apr. 2009.

[35] W.-K. Ma, P.-C. Ching, and B.-N. Vo, "Crosstalk resilient interference cancellation in microphone arrays using capon beamforming," IEEE Trans. Audio Speech Process., vol. 12, no. 5, pp. 468-477, Sep. 2004.

[36] H. Jiang and X. Li, "Application of convex optimization to discriminative training in speech and language processing," IEEE Signal Process. Mag., Special Issue on Convex Optim. for Signal Process., 2010.

[37] T.-H. Chang, Z.-Q. Luo, X. Wu, C. Akgun, J. Vaughan, K. Ugurbil, and P.-F. V. de Moortele, "Transmit B1 shimming at high field with SAR constraints: A two stage optimization independent of the initial set of RF phases and amplitudes," in Proc. 16th Intl. Society for Magnetic Resonance in Medicine, Toronto, Ontario, Canada, May 3-9 2008.

[38] K. W. Cheung, W.-K. Ma, and H. C. So, "Accurate approximation algorithm for TOA-based maximum likelihood mobile location using semidefinite programming," in Proc. IEEE Int. Conf. Acoustics, Speech, and Signal Process., Montreal, Canada, May 2004.

[39] K.-H. Yang, G. Wang, and Z.-Q. Luo, "Efficient convex relaxation methods for robust target localization by a sensor network using time differences of arrivals," IEEE Trans. Signal Process., vol. 57, no. 7, pp. 2775-2784, 2009

[40] R. Zhang, Y.-C. Liang, C.-C. Chai, and S. Cui, "Optimal beamforming for two-way multi-antenna relay channel with analogue network coding," IEEE J. Select. Areas Commun., vol. 27, no. 5, pp. 699-712, 2009.

[41] Z. Quan, W.-K. Ma, S. Cui, and A. Sayed, "Optimal linear fusion for distributed detection via semidefinite programming," to appear in IEEE Trans. Signal Process., 2010.

[42] M. Grant, S. Boyd, and Y. Ye, "CVX: Matlab software for disciplined convex programming," Online, 2009, available: http: / / www . stanford. edu/ boyd/cvx.

[43] Y. Ye, Interior Point Algorithms: Theory and Analysis, ser. WileyInterscience Series in Discrete Mathematics and Optimization. New York: John Wiley \& Sons, Inc., 1997.

[44] C. Helmberg, F. Rendl, R. Vanderbei, and H. Wolkowicz, "An interiorpoint method for semidefinite programming," SIAM J. Optim., vol. 6, no. 2, pp. 342-361, 1996.

[45] W.-K. Ma, C.-C. Su, J. Jaldén, and C.-Y. Chi, "Some results on 16-QAM MIMO detection using semidefinite relaxation," in Proc. IEEE Int. Conf. Acoustics, Speech, and Signal Process., Las Vegas, Nevada, Apr. 2008.
[46] J. F. Sturm, "Using SeDuMi 1.02, a MATLAB toolbox for optimization over symmetric cones," Optim. Meth. Softw., vol. 11-12, pp. 625-653, 1999, software available at http://sedumi.ie.lehigh.edu/.

[47] J. Mattingley and S. Boyd, "Real-time convex optimization in signal processing," IEEE Signal Process. Mag., Special Issue on Convex Optim. for Signal Process., 2010.

[48] L. Vandenberghe and S. Boyd, "Semidefinite programming," SIAM Rev., vol. 38, pp. 49-95, 1996.

[49] M. O. Damen, H. E. Gamal, and G. Caire, "On maximum-likelihood detection and the search for the closest lattice point," IEEE Trans. Inform. Theory, vol. 49, no. 10, pp. 2389-2402, 2003.

[50] S. Verdú, "Computational complexity of optimum multiuser detection," Algorithmica, vol. 4, pp. 303-312, 1989.

[51] J. Jaldén and B. Ottersten, "On the complexity of sphere decoding in digital communications," IEEE Trans. Signal Process., vol. 53, no. 4 pp. 1474-1484, 2005.

[52] A. Wiesel, Y. C. Eldar, and S. Shamai, "Semidefinite relaxation for detection of 16-QAM signaling in MIMO channels," IEEE Signal Process. Lett., vol. 12, no. 9, pp. 653-656, 2005.

[53] N. D. Sidiropoulos and Z.-Q. Luo, "A semidefinite relaxation approach to MIMO detection for higher-order constellations," IEEE Signal Process. Lett., vol. 13, no. 9, pp. 525-528, 2006.

[54] Z. Mao, X. Wang, and X. Wang, "Semidefinite programming relaxation approach for multiuser detection of QAM signals," IEEE Trans. Wireless Commun., vol. 6, no. 12, pp. 4275-4279, 2007.

[55] Y. Yang, C. Zhao, P. Zhou, and W. Xu, "MIMO detection of 16-QAM signaling based on semidefinite relaxation," IEEE Signal Process. Lett., vol. 14, no. 11, pp. 797-800, 2007.

[56] M. Nekuii, M. Kisialiou, T. N. Davidson, and Z.-Q. Luo, "Efficient soft demodulation of MIMO QPSK via semidefinite relxation," in Proc. IEEE Int. Conf. Acoustics, Speech, and Signal Process., Las Vegas, Nevada, Mar. 2008.

[57] J. Jaldén, B. Ottersten, and W.-K. Ma, "Reducing the average complexity of ML detection using semidefinite relaxation," in Proc. IEEE Int. Conf. Acoustics, Speech, and Signal Process., Philadelphia, PA, Mar. 2005.

[58] J. Jaldén and B. Ottersten, "The diversity order of the semidefinite relaxation detector," IEEE Trans. Inform. Theory, vol. 54, no. 4, pp. 1406-1422, Apr. 2008.

[59] M. Kisialiou and Z.-Q. Luo, "Performance analysis of quasi-maximumlikelihood detector based on semi-definite programming," in Proc. IEEE Int. Conf. Acoustics, Speech, and Signal Process., Philadelphia, PA, Mar. 2005.

[60] A. M.-C. So and Y. Ye, "Probabilistic analysis of semidefinite relaxation detectors in multiple-input, multiple-output systems," in Convex Optimization in Signal Processing and Communications, Chapter 5, D. P. Palomar and Y. C. Eldar, Ed., Cambridge University Press, 2010.

[61] N. D. Sidiropoulos, T. N. Davidson, and Z.-Q. Luo, "Transmit beamforming for physical-layer multicasting," IEEE Trans. Signal Process., vol. 54, no. 6, pp. 2239-2251, 2006.

[62] A. M.-C. So, Y. Ye, and J. Zhang, "A unified theorem on SDP rank reduction," Math. Oper. Res., vol. 33, no. 4, pp. 910-920, 2008.

[63] Y. J. Zhang and A. M.-C. So, "Optimal spectrum sharing in MIMO cognitive radio networks via semidefinite programming," Preprint, 2009.

[64] S. Boyd and L. Vandenberghe, Convex Optimization. Cambridge: U.K.: Cambridge University Press, 2004.

[65] M. Bengtsson and B. Ottersten, "Optimal and suboptimal transmit beamforming," in Handbook of Antennas in Wireless Communications, L. C. Godara, Ed., CRC Press, Aug. 2001.

[66] Y. Ye, "Convergence behavior of central paths for convex homogeneous self-dual cones," 1995, unpublished manuscript, available at http: / / www. stanford.edu/ yyye/yyye/ye.ps.

[67] A. Shapiro, "Rank-reducibility of a symmetric matrix and sampling theory of minimum trace factor analysis," Psychometrika, vol. 47, no. 2, pp. 187-199, 1982.

[68] A. I. Barvinok, "Problems of distance geometry and convex properties of quadratic maps," Discr. Comput. Geom., vol. 13, pp. 189-202, 1995.

[69] G. Pataki, "On the rank of extreme matrices in semidefinite programs and the multiplicity of optimal eigenvalues," Math. Oper. Res., vol. 23, no. 2, pp. 339-358, 1998.

[70] W. Ai and S. Zhang, "Strong duality for the CDT subproblem: A necessary and sufficient condition," SIAM J. Optim., vol. 19, no. 4, pp. 1735-1756, 2009.

[71] W. Ai, Y. W. Huang, and S. Zhang, "New results on Hermitian matrix rank-one decomposition," to appear in Math. Program.

[72] A. Beck and Y. C. Eldar, "Regularization in regression with bounded noise: A Chebyshev center approach," SIAM J. Matrix Anal. Appl., vol. 29, no. 2, pp. 606-625, May 2007. 
[73] — "Doubly constrained robust Capon beamformer with ellipsoidal uncertainty sets," IEEE Trans. Signal Process., vol. 55, no. 2, pp. 753 758, Jan. 2007.

[74] Y. C. Eldar, A. Beck, and M. Teboulle, "A minimax Chebyshev estimator for bounded error estimation," IEEE Trans. Signal Process., vol. 56, no. 4, pp. 1388-1397, Apr. 2008.

[75] Y. W. Huang, A. De Maio, and S. Zhang, "Semidefinite programming, matrix decomposition, and radar code design," in Convex Optimization in Signal Processing and Communications, Chapter 6, D. P. Palomar and Y. C. Eldar, Ed., Cambridge University Press, 2010.

[76] J. Jaldén, C. Martin, and B. Ottersten, "Semidefinite programming for detection in linear systems-optimality conditions and space-time coding," in Proc. IEEE Int. Conf. Acoustics, Speech, and Signal Process., Hong Kong, China, Apr. 2003.

[77] Y. T. Chan and K. C. Ho, "A simple and efficient estimator for hyperbolic location,” IEEE Trans. Signal Process., vol. 42, no. 8, pp. 1905-1915, 1994.

[78] K. W. Cheung, H. C. So, W.-K. Ma, and Y. T. Chan, "Least squares algorithms for time-of-arrival-based mobile location," IEEE Trans. Signal Process., vol. 52, no. 4, pp. 1121-1128, 2004.

[79] J. B. Saxe, "Embeddability of weighted graphs in $k$-space is strongly NP-hard," in Proc. 17th Annual Allerton Conf. Communication, Control, and Computing, 1979, pp. 480-489.

[80] A. M.-C. So and Y. Ye, "A semidefinite programming approach to tensegrity theory and realizability of graphs," in Proc. 17th Annual ACM-SIAM Sympos. Discrete Algorithms, Miami, FL, Jan. 2006.

See page ??? (TBD) for the biographies of Zhi-Quan Luo \& Wing-Kin Ma.

Anthony Man-Cho So received his BSE degree in Computer Science from Princeton University in 2000 with minors in Applied and Computational Mathematics, Engineering and Management Systems, and German Language and Culture. He then received his MSc degree in Computer Science in 2002, and his PhD degree in Computer Science with a PhD minor in Mathematics in 2007, all from Stanford University. Dr. So joined the Department of Systems Engineering and Engineering Management at the Chinese University of Hong Kong in 2007. His current research focuses on the interplay between optimization theory and various areas of algorithm design, with applications in portfolio optimization, stochastic optimization, combinatorial optimization, algorithmic game theory, signal processing, and computational geometry.

Yinyu Ye received the B.S. degree in System Engineering from the Huazhong University of Science and Technology, Wuhan, China, and the M.S. and Ph.D. degrees in Management Science \& Engineering from Stanford University, Stanford. Currently, he is a Professor of Management Science and Engineering and Institute of Computational and Mathematical Engineering, and the Director of the $M S \& E$ Industrial Affiliates Program, Stanford University. His current research interests include Continuous and Discrete Optimization, Mathematical Programming, Algorithm Design and Analysis, Computational Game/Market Equilibrium, Metric Distance Geometry, Graph Realization, Dynamic Resource Allocation, and Stochastic and Robust Decision Making, etc.

Shuzhong Zhang received B.Sc. in Applied Mathematics from Fudan University in 1984, and a Ph.D degree in Operations Research and Econometrics from the Tinbergen Institute, Erasmus University, in 1991. He had held faculty positions at Department of Econometrics, University of Groningen (1991-
1993), and Econometric Institute, Erasmus University (19931999). Since 1999, he has been with Department of Systems Engineering \& Engineering Management, The Chinese University of Hong Kong. He received the Erasmus University Research Prize in 1999, the CUHK Vice-Chancellor Exemplary Teaching Award in 2001, the SIAM Outstanding Paper Prize in 2003, and the IEEE Signal Processing Society Best Paper Award. Dr. Zhang is an elected Council Member at Large of the MPS (Mathematical Programming Society) for 2006-2009, and a Vice-President of the Operations Research Society of China. He serves on the Editorial Board of five academic journals, including Operations Research, and SIAM Journal on Optimization. 